\title{
LOCALLY NEARLY SPHERICAL SURFACES ARE ALMOST-POSITIVELY c-CURVED*
}

\author{
PHILIPPE DELANOË ${ }^{\dagger}$ AND YUXIN GE
}

\begin{abstract}
The $c$-curvature of a complete surface with Gauss curvature close to 1 in $C^{2}$ norm is almost-positive (in the sense of Kim-McCann). Our proof goes by a careful case by case analysis combined with perturbation arguments from the constant curvature case, keeping track of an estimate on the closeness curvature condition.
\end{abstract}

Key words. Monge problem, quadratic cost, compact surfaces, nearly spherical, positive $c$ curvature, stability.

AMS subject classifications. Primary 53C21; Secondary 49N60.

1. Introduction and main results. Monge's problem, in optimal transport theory, goes back to [19]. In its general formulation, one is looking for an optimal map $f:(M, \mu) \rightarrow(\bar{M}, \bar{\mu})$ between two Polish probability spaces. The optimality criterion consists in minimizing the total cost functional $\int_{M} c(x, f(x)) d \mu(x)$ among measurable maps which push $\mu$ to $\bar{\mu}$, where the cost function $c: M \times \bar{M} \rightarrow \mathbb{R} \cup\{+\infty\}$ is given lower semi-continuous with some additional properties (see e.g. [21] and references therein). In the emblematic case of the Brenier-McCann cost function: $M=\bar{M}, c=\frac{1}{2} d^{2}$, where $M$ stands for a complete Riemannian manifold with associated distance function $d$, this problem was solved under mild assumptions on the given probability measures $\mu$ and $\bar{\mu}[2,17]$. In that case, the optimal map must read $f=\exp (\operatorname{grad} u)$ for some $c$-convex potential function $u$ such that the pushing condition $f_{\#} \mu=\bar{\mu}$ becomes a partial differential equation of Monge-Ampère type satisfied by $u$ in a weak sense. Neil Trudinger and his co-workers observed that a similar solution scheme exists for a class of more general cost functions $c$ for which, given smooth data, they analyzed the smoothness of the corresponding potential function $u$ [16]. For the purpose of a one-sided interior estimate on an expression of second order (in $u$ ), they were lead to formulate a fourth-order two-points condition on the cost function $c$, called (A3S) condition. A weak form of the latter, called (A3W), was proved necessary (for the smoothness of $u$ ) by Loeper [14]; in particular, in the Brenier-McCann case, he interpreted (A3W) read on the diagonal of $M \times M$ as the non-negativity of the sectional curvature of $M$. Lately, still with $c=\frac{1}{2} d^{2}$, Cédric Villani and his co-workers were able to relate some variants of (A3S), checked stable at round spheres under $C^{4}$ small deformations of the standard round metric, with the convexity of the tangential domain of injectivity of the exponential map $[15,8,10]$. However, the very geometrical status of the fourth-order expression (in c) occuring in condition (A3S) was not understood untill Kim and McCann interpreted it [11] as a genuine, though quite special, curvature expression arising on the product manifold $M \times \bar{M}$ endowed with the pseudo-Riemannian metric:

\footnotetext{
*Received September 18, 2010; accepted for publication August 9, 2011.

†Université de Nice-Sophia Antipolis, Faculté des Sciences, Laboratoire J.-A. Dieudonné, Parc Valrose, F-06108 Nice Cedex 2, France (Philippe.DELANOE@unice.fr). Supported by the CNRS.

$\ddagger$ Université Paris-Est Créteil Val de Marne, Faculté des Sciences et Technologie, Centre de Mathématiques, 61 avenue du Général De Gaulle, F-94010 Créteil Cedex, France (ge@univparis12.fr).
} 
$h=-\frac{1}{2} \frac{\partial^{2} c}{\partial x^{i} \partial \bar{x}^{j}}\left(d x^{i} \otimes d \bar{x}^{j}+d \bar{x}^{j} \otimes d x^{i}\right)$. They also defined an extended version of (A3S), stronger than (A3W), called non-negative cross-curvature condition (NNCC, for short ${ }^{1}$ ) and proved that it is stable under Cartesian product ${ }^{2}$ as well as, in the Brenier-McCann case, under Riemannian submersion [12]. Actually, in that case, they defined a stronger condition called almost-positive cross-curvature condition (APCC, for short ${ }^{3}$ ) also shown stable under Riemannian submersion [12]. So, with $c=\frac{1}{2} d^{2}$, the stability of NNCC (resp. APCC) under products (resp. submersions) enables to construct new NNCC (resp. APCC) examples out of known ones - like the standard sphere [12]. In the present paper, we will prove the stability of APCC at the standard 2-sphere; specifically, we will check the APCC condition for $c=\frac{1}{2} d^{2}$ on a complete surface with Gauss curvature $C^{2}$ close to a positive constant. This result complements the stability one of [8] on the 2 -sphere as well as an unstated one (stability of APCC at the standard $n$-sphere, near conjugacy, see Remark 1 below) obtained in the course of a proof in [10]. Here, let us point out that our paper is drawn from an initial 44 pages draft sent by Ge to Figalli in January 2009, thus independent from the papers $[9,10]$ first circulated in July 2009; in particular, our analysis of the APCC property near conjugacy (Section 4 below) departs from that of [10].

In order to state our result, let us first recall some definitions, restricting to connected complete Riemannian manifolds $M=\bar{M}$ with the cost function $c=\frac{1}{2} d^{2}$ defined on $M \times \bar{M} \backslash$ Cut, where Cut stands for the cut locus. Using the aforementioned pseudo-Riemannian metric $h$ on $M \times \bar{M}$ and setting $\operatorname{Sect}_{h}$ for its sectional curvature tensor viewed as a field of quadratic forms on $\bigwedge^{2} T(M \times \bar{M})$, for each $(m, \bar{m}) \in$ $M \times \bar{M} \backslash$ Cut and each $(\xi, \bar{\xi}) \in T_{m} M \times T_{\bar{m}} \bar{M}$, the associated cross-curvature is defined by [11]:

$$
\operatorname{cross}_{(m, \bar{m})}(\xi, \bar{\xi}):=\operatorname{Sect}_{h}[(\xi \oplus 0) \wedge(0 \oplus \bar{\xi})] .
$$

Kim and McCann observed that it must vanish for some choice of $(\xi, \bar{\xi})$ [12]. If it is identically non-negative, we say that the manifold $M$ is NNCC. For instance, the standard $n$-sphere is NNCC $[12,8]$ and if a manifold $M$ endowed with a Riemannian metric $g$ is so, its sectional curvature tensor Sect $_{g}$ must be non-negative because, at $\bar{m}=m$, we have: $\operatorname{cross}_{(m, m)}(\xi, \bar{\xi}) \equiv \frac{4}{3} \operatorname{Sect}_{g}(\xi \wedge \bar{\xi})$, as first observed by Loeper [14]. Pulling back by the exponential map:

$$
(m, V) \in \mathrm{NoCut} \longrightarrow\left(m, \exp _{m}(V)\right) \in M \times \bar{M} \backslash \mathrm{Cut},
$$

where NoCut is the domain of $T M$ defined by:

$$
\text { NoCut : }=\left\{(m, V) \in T M, \forall t \in[0,1], \exp _{m}(t V) \notin \mathrm{Cut}_{m}\right\}
$$

(and $\mathrm{Cut}_{m}$, the cut locus of the point $m$ ), Trudinger et al noted [16, p.164] that one identically recovers $\frac{1}{2} \operatorname{cross}_{(m, \bar{m})}(\xi, \bar{\xi})$ at $\bar{m}=\exp _{m}(V)$ with $(m, V) \in$ NoCut and $\bar{\xi}=d\left(\exp _{m}\right)(V)(\nu)$, by calculating the quantity:

$$
\mathcal{C}(m, V)(\xi, \nu):=-\frac{D^{2}}{d \lambda^{2}}[A(m, V+\lambda \nu)(\xi)]_{\lambda=0}
$$

\footnotetext{
${ }^{1}$ also used below as an abbreviation for non-negatively cross-curved

${ }^{2}$ unlike (A3S), or even (A3W) alone as soon as a factor is not NNCC

3 also used below as an abbreviation for almost-positively cross-curved
} 
where: $A(m, V)(\xi):=\nabla d\left[p \rightarrow c\left(p, \exp _{m}(V)\right)\right]_{p=m}(\xi, \xi)$ with $\nabla$ the Levi-Civita connection of the Riemannian metric $g$ and where $D$ stands for the canonical flat connection of $T_{m} M$. In [7], we performed a stepwise calculation of $A(m, V)(\xi)$ and its first and second derivatives with respect to $V$, in a Fermi chart along the geodesic $t \in[0,1] \rightarrow \exp _{m}(t V) \in M$. This calculation just requires that $(m, V)$ belong to NoConj, denoting so the domain ${ }^{4}$ of $T M$ which consists of tangent vectors $(m, W) \in T M$ such that the geodesic segment $t \in[0,1] \rightarrow \exp _{m}(t W)$ contains no conjugate points, a fact conceptualized in [8] using the Hamiltonian flow (see also [10]). Neil Trudinger suggested that one calls the quantity $\mathcal{C}(m, V)(\xi, \nu)$ defined by (1), now with $(m, V) \in$ NoConj, the $c$-curvature ${ }^{5}$ of $M$ at $(m, V, \xi, \nu)$. It is known to vanish if $\operatorname{rank}(V, \xi, \nu) \leq 1[7,12]$. Now, the definition given in [12] of an APCC (resp. NNCC) manifold reads in terms of the $c$-curvature as follows:

Definition 1. Let $M$ be a connected complete Riemannian manifold with cost function $c=\frac{1}{2} d^{2}$. We say that $M$ is non-negatively $c$-curved, or NNCC, if

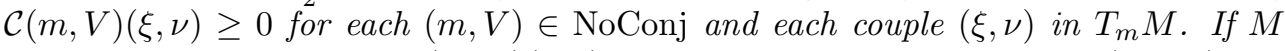
is $N N C C$ and such that: $\mathcal{C}(m, V)(\xi, \nu)=0$ if and only if the span of $(V, \xi, \nu)$ has dimension at most 1 , we call it almost-positively c-curved, or APCC.

REMARK 1. An intermediate (unquantified) result of [10], unstated there as such, obtained via the square completion of a huge expression, goes as follows:

If $M$ is the $n$-sphere endowed with a Riemannian metric $C^{4}$ close to the standard one and if $(m, V) \in$ NoConj lies close enough to the boundary of NoConj, then $(\xi, \nu) \mapsto$ $\mathcal{C}(m, V)(\xi, \nu)$ satisfies the $A P C C$ property on $T_{m} M \times T_{m} M$.

Let us call, for short, a surface any smooth compact connected 2-dimensional Riemannian manifold without boundary. We aim at the following result:

TheOREM 1. Let $S$ be a surface with Gauss curvature $K$ such that $\min _{S} K=1$. There exists a small universal constant $\eta>0$ such that, if $|K-1|_{C^{2}(S)} \leq \eta$, then $S$ is $A P C C$.

Here, the $C^{2}$ norm of a function $f: S \rightarrow \mathbb{R}$ is defined (using the Riemannian norm $|$.$| on tensors) by: |f|_{C^{2}(S)}:=\sup _{S}|f|+\sup _{S}|d f|+\sup _{S}|\nabla d f|$.

The result is proved in [12] with $\eta=0$ (constant curvature case, see also [8] for NNCC) and in [10] with $(m, V) \in$ NoConj lying close enough to the boundary of NoConj (with no quantified estimates, though). If $V=0$, the result is obvious (due to the cross-curvature interpretation when $m=\bar{m}$ ), so we will assume $V \neq 0$ with no loss of generality.

REMARK 2. Let

$$
\mathrm{D}_{c}=\sup \left\{|V|_{m}, \quad(m, V) \in \mathrm{NoConj}\right\}
$$

be the diameter of conjugacy of $S$. Since $K \geq 1$, the Bonnet-Myers theorem $[1,4,6$, 18] implies: $\mathrm{D}_{c} \leq \pi$; in particular, the diameter of $S$ must be at most equal to $\pi$.

Actually, we will prove a stronger result, namely:

\footnotetext{
${ }^{4}$ as well-known $[4,6]$, NoConj is the maximum rank domain for the exponential map which contains NoCut

${ }^{5}$ somewhat consistently with the $c$-segment denomination used in [16]; we will use this short denomination, instead of 'extended MTW tensor' as in [15, 9, 10] or 'Ma-Trudinger-Wang curvature' as in $[9,13]$, since further names could be associated to the birth of this conceptual object, anyhow
} 
THEOREM 2. Let $S$ be a surface with $\min _{S} K=1$. There exists small universal positive constants $\eta, \varsigma$ such that, if $|K-1|_{C^{2}(S)} \leq \eta$, for any $(m, V) \in$ NoConj and any couple $(\xi, \nu)$ of unit vectors in $T_{m} S$, the following inequality holds:

$$
\mathcal{C}(m, V)(\xi, \nu) \geq \varsigma \mathcal{A}_{2}(m, V, \xi, \nu),
$$

where $\mathcal{A}_{2}(m, V, \xi, \nu)$ stands for the sum of the squared areas of the parallelograms repectively defined in $T_{m} S$ by the couples $(\xi, \nu),(V, \xi),(V, \nu)$, in other words:

$$
\mathcal{A}_{2}(m, V, \xi, \nu)=|\xi|^{2}|\nu|^{2}-g(\xi, \nu)^{2}+|V|^{2}|\xi|^{2}-g(V, \xi)^{2}+|V|^{2}|\nu|^{2}-g(V, \nu)^{2} .
$$

The outline of the paper essentially coincides with that of the proof. We present a quick derivation of the $c$-curvature expression in Section 2 and related perturbative estimates for that expression, based on the assumption that the $C^{2}$ norm of $(K-1)$ is small, in Section 3. Using the latter, we prove successively Theorem 2 under the additional assumption that the point $\exp _{m}(V)$ lies, either near the first conjugate point $m^{*}$ of $m$ along the geodesic $t \in \mathbb{R}^{+} \rightarrow \exp _{m}(t V) \in S$ (Section 4 ), or near $m$ (Section 5), or in-between (Section 6). The proof of Theorem 2 itself, as a whole, is provided in Section 7, by synthetizing the various, sometimes redundant, smallness assumptions made in the previous sections on $|K-1|_{C^{2}(S)}$, $\varsigma$ and an extra parameter $\delta$ used to locate $\exp _{m}(V)$ with respect to $m$ and $m^{*}$ as just described. The proof of the main perturbation lemma is deferred to Appendix A, but Section 3 includes a straightforward application of it to a uniform convexity estimate for the boundary of NoConj.

Finally, a warning must be made about some notations and conventions used below. Starting from Lemma 1 (Section 3), we will abbreviate $|K-1|_{C^{2}(S)}$ merely by $\varepsilon$. In Section 4 (resp. Section 5 ), we will set $\delta_{1} d\left(m, m^{*}\right)$ (resp. $\delta_{2}$ ) for the maximal distance assumed between $\exp _{m}(V)$ and the first conjugate point $m^{*}$ (resp. and the point $m$ ); consistently in Section 6 , we will set $\frac{1}{2} \delta_{1} d\left(m, m^{*}\right)$ (resp. $\left.\frac{1}{2} \delta_{2}\right)$ for the minimal distance at which $\exp _{m}(V)$ must stay away from $m^{*}$ (resp. from $m$ ) on that geodesic. In the course of the proof, starting from Lemma 1, we will require various (fairly explicit, universal) smallness conditions on $\varepsilon$ or the auxiliary position parameters $\delta_{i}$ 's. Furthermore, in each case or subcase distinguished below for $(m, V, \xi, \nu)$, we will find a different value of the (small positive) constant $\varsigma$ occuring in (2); the actual value to be taken for $\varsigma$ in the statement of Theorem 2 will be, of course, the smallest among them. The various universal ${ }^{6}$ constants and smallness conditions arising in the paper are listed in Appendix B to which the reader should systematically refer.

2. c-curvature expression in dimension 2. Henceforth, we fix a surface $S$, a point $m_{0} \in S$ and three non-zero tangent vectors $\left(V_{0}, \xi, \nu\right)$ in $T_{m_{0}} S$ with $\left(m_{0}, V_{0}\right) \in$ NoCut and $(\xi, \nu)$ linearly independent. We wish to calculate the $c$ curvature $\mathcal{C}\left(m_{0}, V_{0}\right)(\xi, \nu)$.

2.1. General case. A chart $x=\left(x^{1}, x^{2}\right)$ of $S$ centered at $m_{0}$ such that the local components $g_{i j}(x)$ of the metric satisfy: $g_{i j}(0)=\delta_{i j}, d g_{i j}(0)=0$, is called normal at $m_{0}$; let $x$ be such a chart. We set $v=\left(v^{1}, v^{2}\right)$ for the fiber coordinates of $T S \rightarrow S$ naturally associated to $x$, use Einstein's convention and abbreviate partial derivatives as follows:

$$
\partial_{i}=\frac{\partial}{\partial x^{i}}, \partial_{i j}=\frac{\partial^{2}}{\partial x^{i} \partial x^{j}}, \ldots ; D_{i}=\frac{\partial}{\partial v^{i}}, D_{i j}=\frac{\partial^{2}}{\partial v^{i} \partial v^{j}}, \ldots
$$

\footnotetext{
${ }^{6}$ thus, in particular, independent of $(m, V) \in$ NoConj
} 
For each $(m, V) \in$ NoCut with $m$ in the domain of the chart $x$, we set:

$$
X=X(x, v, t)=\left(X^{1}(x, v, t), X^{2}(x, v, t)\right)=x\left(\exp _{m}(t V)\right),
$$

where $x=x(m)$ and $V=v^{i} \partial_{i}$. For $V \in T_{m_{0}} S$ such that $\left(m_{0}, V\right) \in$ NoCut, and setting $\xi=\xi^{i} \partial_{i}$, we recall from [7] that the quadratic form $A\left(m_{0}, V\right)(\xi)$ defined in the introduction is equal to $A_{i j}(v) \xi^{i} \xi^{j}$ with:

$$
A_{i j}(v)=Y_{k}^{i}(v) \partial_{j} X^{k}(0, v, 1)
$$

and the matrix $Y_{k}^{i}(v)$ given by: $Y_{k}^{i}(v) D_{j} X^{k}(0, v, 1)=\delta_{j}^{i}$. Given $V=v^{i} \partial_{i}$ as above, it is convenient to compute the right-hand side of (3) by choosing for $x$ a particular normal chart at $m_{0}$ (unique up to $x^{1} \rightarrow-x^{1}$ ), namely:

Definition 2. A Fermi chart along $V$ is a normal chart $x$ at $m_{0}$ such that $V=r \partial_{2}$ (with $r=|V|$ ) and the Riemannian metric reads:

$$
g=d x^{1} \otimes d x^{1}+G\left(x^{1}, x^{2}\right) d x^{2} \otimes d x^{2}, \text { with } G\left(0, x^{2}\right)=1, \partial_{1} G\left(0, x^{2}\right)=0 .
$$

Let $x$ be a Fermi chart along $V$. The geodesic $t \in[0,1] \rightarrow m_{t}=\exp _{m}(t V) \in S$ (called the axis of the chart) simply reads $t \mapsto X((0,0),(0, r), t)=(0, t r)$ and, for fixed $x^{2}$, the paths which read $t \mapsto\left(t, x^{2}\right)$ are geodesics of $S$ as well, orthogonal to the axis. The Christoffel symbols are given by:

$$
\Gamma_{22}^{1}=-\frac{1}{2} \partial_{1} G, \Gamma_{12}^{2}=\frac{\partial_{1} G}{2 G}, \Gamma_{22}^{2}=\frac{\partial_{2} G}{2 G}, \text { others vanish }
$$

and the Gauss curvature, by $K=-\frac{\partial_{11} \sqrt{G}}{\sqrt{G}}$. We thus get for the derivatives of the Christoffel symbols on the axis, intrinsic expressions given in terms of $K$ at $x=\left(0, x^{2}\right)$ by:

$$
\partial_{1} \Gamma_{22}^{1}=-\partial_{1} \Gamma_{12}^{2}=K, \partial_{11} \Gamma_{22}^{1}=-\partial_{11} \Gamma_{12}^{2}=\partial_{1} K, \partial_{1} \Gamma_{22}^{2}=0, \partial_{11} \Gamma_{22}^{2}=-\partial_{2} K .
$$

With these formulas at hand, we readily find:

$$
\partial X((0,0),(0, r), t)=\left(\begin{array}{cc}
f_{0}(t) & 0 \\
0 & 1
\end{array}\right), D X((0,0),(0, r), t)=\left(\begin{array}{cc}
f_{1}(t) & 0 \\
0 & t
\end{array}\right)
$$

where $f_{i}(t)=f_{i}((0,0),(0, r), t)$ for $i \in\{0,1\}$; here, $f_{i}(x, w, t)$ are the expressions in the chart $x$ of the solutions for $t \in[0,1]$ of the Jacobi equation:

$$
\ddot{f}+|W|^{2} K\left(\exp _{m}(t W)\right) f=0
$$

(where $x=x(m), W=w^{i} \partial_{i}$ with $(m, W) \in$ NoConj, and we use the dot notation: $\left.\dot{f}=\frac{d f}{d t}, \ddot{f}=\frac{d^{2} f}{d t^{2}}\right)$, satisfying the initial condition:

$$
f_{i}(0)=\delta_{i 0}, \dot{f}_{i}(0)=\delta_{i 1}
$$


REMARK 3. For later use, we observe that, for $t \in(0,1]$ and $(m, W) \in$ NoConj, we have: $0<f_{1}(x, w, t)$. Moreover, Sturm comparison theorem [4] combined with Remark 2 provides the pinching:

$$
\frac{\sin \left(\sqrt{\max _{S} K}|W| t\right)}{\sqrt{\max _{S} K}|W|} \leq f_{1}(x, w, t) \leq \frac{\sin (|W| t)}{|W|},
$$

which yields $f_{1}(x, w, t) \leq t \leq 1$ and $\lim _{|W| \downarrow 0} f_{1}(x, w, 1)=1$.

Back to $(m, W)=\left(m_{0}, V\right)$, applying $(3)$ in our Fermi chart along $V$, we get:

$$
A\left(m_{0}, V\right)(\xi)=|\xi|^{2}-\left(1-\frac{f_{0}(1)}{f_{1}(1)}\right)|\xi-g(\xi, U) U|^{2}, \text { with } U=\frac{V}{|V|} .
$$

Here comes a key observation, also made in [9] (and extended to the higher dimensional setting in [10], see also [13]): the right-hand side of the preceding equation is intrinsic because so is (4). We may thus use a single Fermi chart $x$, along the sole tangent vector $V_{0}$ at $m_{0}$, and write for each $V=v^{i} \partial_{i} \in T_{m_{0}} S$ close to $V_{0}$ :

$$
A\left(m_{0}, V\right)(\xi)=|\xi|^{2}-\left(1-\frac{f_{0}(0, v, 1)}{f_{1}(0, v, 1)}\right)|\xi-g(\xi, U) U|^{2} .
$$

We will now calculate the $c$-curvature $\mathcal{C}\left(m_{0}, V_{0}\right)(\xi, \nu)$ in that Fermi chart (fixed once for all), by combining (1) with (5). Letting henceforth $\xi$ and $\nu$ be unit vectors and orienting the tangent plane $T_{m_{0}} S$ by the local volume form $d x^{1} \wedge d x^{2}$, we denote by $\vartheta($ resp. $\varphi)$ the angle in $[0,2 \pi)$ by which a direct rotation brings $\xi$ (resp. $\nu$ ) to $U_{0}=\frac{V_{0}}{\left|V_{0}\right|}=\partial_{2}$; in other words, we set:

$$
\xi=\sin \vartheta \partial_{1}+\cos \vartheta \partial_{2}, \nu=\sin \varphi \partial_{1}+\cos \varphi \partial_{2} .
$$

A lengthy but routine calculation yields:

$$
\begin{aligned}
\mathcal{C}\left(m_{0}, V_{0}\right)(\xi, \nu)= & -\sin ^{2} \vartheta\left(\frac{f_{0}^{\prime \prime}}{f_{1}}-\frac{f_{0} f_{1}^{\prime \prime}}{f_{1}^{2}}-\frac{2 f_{0}^{\prime} f_{1}^{\prime}}{f_{1}^{2}}+\frac{2 f_{0}\left(f_{1}^{\prime}\right)^{2}}{f_{1}^{3}}\right) \\
& +\frac{2}{r_{0}^{2}}\left(\cos ^{2} \vartheta-\cos ^{2}(\vartheta+\varphi)\right)\left(1-\frac{f_{0}}{f_{1}}\right) \\
& +\frac{4}{r_{0}} \cos \vartheta \sin \vartheta \sin \varphi\left(\frac{f_{0}^{\prime}}{f_{1}}-\frac{f_{0} f_{1}^{\prime}}{f_{1}^{2}}\right),
\end{aligned}
$$

where we have set, for short: $f_{a}^{\prime}=\nu^{i} D_{i} f_{a}\left(0, v_{0}, 1\right), f_{a}^{\prime \prime}=\nu^{i} \nu^{j} D_{i j} f_{a}\left(0, v_{0}, 1\right)$, for $a=0,1$, and $v_{0}=\left(0, r_{0}\right)$ with $r_{0}=\left|V_{0}\right|$.

2.2. Constant curvature case recalled. Setting for short $\kappa=K\left(m_{0}\right)$ and $\bar{r}=\sqrt{\kappa} r$, let us recall the expressions which occur for $f_{0}, f_{1}$ in case $K \equiv \kappa$, labelling them all with a bar:

$$
\bar{f}_{0}(0, v, t)=\cos (\bar{r} t), \bar{f}_{1}(0, v, t)=\frac{\sin (\bar{r} t)}{\bar{r}}, \text { where } r=\sqrt{\left(v^{1}\right)^{2}+\left(v^{2}\right)^{2}} .
$$

At $(v, t)=\left(v_{0}, 1\right)$, with $v_{0}=\left(0, r_{0}\right)$ and $\bar{r}_{0}=\sqrt{\kappa} r_{0}$, we infer correspondingly:

$$
\bar{f}_{0}^{\prime}=-\sqrt{\kappa} \sin \bar{r}_{0} \cos \varphi, \quad \bar{f}_{0}^{\prime \prime}=\kappa\left(-\frac{\sin \bar{r}_{0}}{\bar{r}_{0}}+\left(\frac{\sin \bar{r}_{0}}{\bar{r}_{0}}-\cos \overline{r_{0}}\right) \cos ^{2} \varphi\right)
$$




$$
\begin{gathered}
\bar{f}_{1}^{\prime}=\frac{\sqrt{\kappa}}{\bar{r}_{0}}\left(\cos \bar{r}_{0}-\frac{\sin \bar{r}_{0}}{\bar{r}_{0}}\right) \cos \varphi, \\
\bar{f}_{1}^{\prime \prime}=\frac{\kappa}{\bar{r}_{0}^{2}}\left(\cos \bar{r}_{0}-\frac{\sin \bar{r}_{0}}{\bar{r}_{0}}+\left(3\left(\frac{\sin \bar{r}_{0}}{\bar{r}_{0}}-\cos \bar{r}_{0}\right)-\bar{r}_{0} \sin \bar{r}_{0}\right) \cos ^{2} \varphi\right),
\end{gathered}
$$

hence:

$$
\begin{aligned}
\frac{1}{\kappa} \overline{\mathcal{C}}\left(m_{0}, V_{0}\right)(\xi, \nu) & =\sin ^{2} \vartheta \sin ^{2} \varphi \frac{\bar{r}_{0}^{2}+\bar{r}_{0} \cos \bar{r}_{0} \sin \bar{r}_{0}-2 \sin ^{2} \bar{r}_{0}}{\bar{r}_{0}^{2} \sin ^{2} \bar{r}_{0}} \\
& +2 \sin ^{2} \vartheta \cos ^{2} \varphi \frac{\sin \bar{r}_{0}-\bar{r}_{0} \cos \bar{r}_{0}}{\sin ^{3} \bar{r}_{0}} \\
& +2 \cos ^{2} \vartheta \sin ^{2} \varphi \frac{\sin \bar{r}_{0}-\bar{r}_{0} \cos \bar{r}_{0}}{\bar{r}_{0}^{2} \sin \bar{r}_{0}} \\
& +4 \cos \vartheta \sin \vartheta \cos \varphi \sin \varphi \frac{\sin ^{2} \bar{r}_{0}-\bar{r}_{0}^{2}}{\bar{r}_{0}^{2} \sin ^{2} \bar{r}_{0}}
\end{aligned}
$$

3. Perturbative tools. In the sequel of the paper, dropping the first argument $x=x(m)$ since it is fixed, equal to $(0,0)=x\left(m_{0}\right)$, we simply write: $f_{a}=f_{a}(v, t), X=$ $X(v, t)$ and, abusively with the same letter: $K(X(v, t))=K\left(\exp _{m_{0}}(t V)\right)$, where $V=v^{i} \partial_{i}$. Moreover, anytime the second argument $v$ is equal to $v_{0}=\left(0, r_{0}\right)$, we will also drop it and just write: $f_{a}=f_{a}(t)$ and so on.

Given a real number $\omega>0$, we will require the linear map:

$$
f \in C^{0}([0,1], \mathbb{R}) \longrightarrow \mathcal{S}_{\omega}(f) \in C^{0}([0,1], \mathbb{R})
$$

defined as the solution map $f \mapsto u$ of the linear initial value problem:

$$
\ddot{u}+\omega^{2} u=f, u(0)=\dot{u}(0)=0 .
$$

The representation formula: $\mathcal{S}_{\omega}(f)(t)=\int_{0}^{t} \frac{\sin (\omega(t-\tau))}{\omega} f(\tau) d \tau$ is well known. Setting $\|v\|=\sup _{t \in[0,1]}|v(t)|$, it yields for $\mathcal{S}_{\omega}$ the contraction estimate:

$$
\left\|\mathcal{S}_{\omega}(f)\right\| \leq \frac{1}{2}\|f\|
$$

easily obtained by writing:

$$
u(t)=\int_{0}^{t} \dot{u}(\tau) d \tau=\int_{0}^{t} \int_{0}^{\tau} \cos (\omega(\tau-\theta)) f(\theta) d \theta d \tau .
$$

We will also require the following formulas (written at $t=1$, for $f(t)=t$ and $f(t)=$ $\left.t^{2}\right)$ :

$$
\mathcal{S}_{\bar{r}_{0}}(t)(1)=\frac{\bar{r}_{0}-\sin \bar{r}_{0}}{\bar{r}_{0}^{3}}, \mathcal{S}_{\bar{r}_{0}}\left(t^{2}\right)(1)=\frac{\bar{r}_{0}^{2}+2\left(\cos \bar{r}_{0}-1\right)}{\bar{r}_{0}^{4}} .
$$

We are now ready to state our main perturbation lemma, the proof of which is deferred to Appendix A: 
Lemma 1. If $|K-1|_{C^{2}(S)} \leq \frac{1}{\pi^{2}}$, there exists universal constants $B_{1 k a}, B_{2 k a}, B_{3 k a}$, for $a \in\{0,1\}$ and $k \in\{0,1,2\}$, such that the following estimates hold:

$$
\begin{gathered}
\left\|D_{\nu}^{k} f_{a}\right\| \leq B_{1 k a},\left\|D_{\nu}^{k}\left(f_{a}-\bar{f}_{a}\right)\right\| \leq B_{2 k a} \varepsilon r_{0}^{2-k}, \\
\left\|D_{\nu}^{k}\left(f_{a}-\bar{f}_{a}\right)+r_{0}^{3-k} \psi_{k} \mathcal{S}_{\bar{r}_{0}}\left(t^{a+1}\right)\right\| \leq B_{3 k a} \varepsilon r_{0}^{4-k},
\end{gathered}
$$

where, for short, $\varepsilon:=|K-1|_{C^{2}(S)}$ and:

$$
\begin{gathered}
\psi_{0}:=\partial_{2} K(0), \psi_{1}:=3 \cos \varphi \partial_{2} K(0)+\sin \varphi \partial_{1} K(0), \\
\psi_{2}:=\left(2+4 \cos ^{2} \varphi\right) \partial_{2} K(0)+4 \sin \varphi \cos \varphi \partial_{1} K(0)
\end{gathered}
$$

(from now on, we will freely use to these abbreviations).

REMARK 4. Let us stress that the bounds:

$$
\forall a=0,1,\left\|D_{12} f_{a}\right\| \leq 2 B_{12 a},\left\|D_{12}\left(f_{a}-\bar{f}_{a}\right)\right\| \leq 2 B_{22 a} \varepsilon,
$$

follow from thoses on $\left\|D_{\nu \nu} f_{a}\right\|$ and $\left\|D_{\nu \nu}\left(f_{a}-\bar{f}_{a}\right)\right\|$ by letting $\nu=\frac{1}{\sqrt{2}}\left(\partial_{1}+\partial_{2}\right)$.

The first line of conclusion of Lemma 1 will be used to prove Theorem 1 near $^{7}$ the first conjugate point (Section 4 ). Uniformly away from that point, and crucially for $r_{0} \downarrow 0$, the proof requires the second line of conclusion through a Maclaurin type approximation estimate for the $c$-curvature, namely:

Corollary 1. If $|K-1|_{C^{2}(S)} \leq \frac{1}{\pi^{2}}$ and $\bar{r}_{0}<\pi$, there exists a universal constant $C_{1}$ such that the absolute value of the following expression:

$$
\begin{aligned}
\frac{f_{1}^{3}}{\bar{f}_{1}^{3}} \mathcal{C}\left(m_{0}, V_{0}\right)(\xi, \nu) & -\overline{\mathcal{C}}\left(m_{0}, V_{0}\right)(\xi, \nu)-\frac{r_{0} \psi_{2} \sin ^{2} \vartheta}{\bar{f}_{1}}\left(\mathcal{S}_{\bar{r}_{0}}(t)(1)-\frac{\bar{f}_{0} \mathcal{S}_{\bar{r}_{0}}\left(t^{2}\right)(1)}{\bar{f}_{1}}\right) \\
& +\frac{2 r_{0} \psi_{0} \mathcal{S}_{\bar{r}_{0}}\left(t^{2}-t\right)(1)}{\bar{f}_{1}}\left(\cos ^{2} \vartheta-\cos ^{2}(\vartheta+\varphi)\right) \\
& +\frac{4 r_{0} \psi_{1} \cos \vartheta \sin \vartheta \sin \varphi}{\bar{f}_{1}}\left(\mathcal{S}_{\bar{r}_{0}}(t)(1)-\frac{\bar{f}_{0} \mathcal{S}_{\bar{r}_{0}}\left(t^{2}\right)(1)}{\bar{f}_{1}}\right)
\end{aligned}
$$

is bounded above by:

$$
\frac{1}{\bar{f}_{1}^{3}} C_{1}^{3} \pi^{8} \varepsilon r_{0}^{2}\left(338 \sin ^{2} \vartheta+268 \cos ^{2} \vartheta \sin ^{2} \varphi\right) .
$$

Proof of the corollary. For each $a \in\{0,1\}$ and $k \in\{0,1,2\}$, we split $D_{\nu}^{k} f_{a}$ identically into three summands: $D_{\nu}^{k} f_{a}=S_{1}^{(k, a)}+S_{2}^{(k, a)}+S_{3}^{(k, a)}$ given by:

$$
S_{1}^{(k, a)}=D_{\nu}^{k} \bar{f}_{a}, S_{2}^{(k, a)}=-r_{0}^{3-k} \psi_{k} \mathcal{S}_{\bar{r}_{0}}\left(t^{a+1}\right)(1) .
$$

From (9), we define the constants $c_{6}, c_{7}$ as in Appendix B. From Lemma 1, we know that

$$
\left|S_{1}^{(k, a)}\right| \leq B_{1 k a},\left|S_{3}^{(k, a)}\right| \leq B_{3 k a} \varepsilon r_{0}^{4-k}
$$

\footnotetext{
${ }^{7}$ where $\overline{\mathcal{C}}\left(m_{0}, V_{0}\right)(\xi, \nu)$ could blow up since $\bar{r}_{0}$ could exit from $(0, \pi)$ for $\varepsilon \neq 0$
} 
and from the obvious bounds:

$$
\left|\psi_{0}\right| \leq \varepsilon,\left|\psi_{1}\right| \leq 4 \varepsilon,\left|\psi_{2}\right| \leq 8 \varepsilon
$$

we further know that

$$
\left|S_{2}^{(k, a)}\right| \leq 8 c_{6+a} \varepsilon r_{0}^{3-k}
$$

Let us consider the expression (6) of the $c$-curvature, multiply it by $f_{1}^{3}$ and, using the preceding splittings and bounds, let us estimate the Maclaurin approximation of each of the three auxiliary expressions:

$$
\begin{gathered}
E_{1}:=f_{1}^{2} f_{0}^{\prime \prime}-f_{0} f_{1} f_{1}^{\prime \prime}-2 f_{1} f_{0}^{\prime} f_{1}^{\prime}+2 f_{0}\left(f_{1}^{\prime}\right)^{2} \\
E_{2}:=\frac{2}{r_{0}^{2}} f_{1}^{2}\left(f_{1}-f_{0}\right), E_{3}:=\frac{4}{r_{0}} f_{1}\left(f_{1} f_{0}^{\prime}-f_{0} f_{1}^{\prime}\right),
\end{gathered}
$$

which occur in $f_{1}^{3} \mathcal{C}\left(m_{0}, V_{0}\right)(\xi, \nu)$ as coefficients, respectively of:

$$
-\sin ^{2} \vartheta,\left(\cos ^{2} \vartheta-\cos ^{2}(\vartheta+\varphi)\right), \cos \vartheta \sin \vartheta \sin \varphi
$$

Setting $\bar{E}_{1}, \bar{E}_{2}, \bar{E}_{3}$, for the corresponding quantities defined with $\bar{f}_{0}, \bar{f}_{1}$ instead of $f_{0}, f_{1}$, and proceeding stepwise, with careful intermediate calculations ${ }^{8}$, we get for the $\left(E_{\ell}-\bar{E}_{\ell}\right)$ 's the following analogues of the second line of conclusion of Lemma 1 :

$$
\begin{gathered}
\left|E_{1}-\bar{E}_{1}+r_{0} \psi_{2} \bar{f}_{1}\left[\bar{f}_{1} \mathcal{S}_{\bar{r}_{0}}(t)(1)-\bar{f}_{0} \mathcal{S}_{\bar{r}_{0}}\left(t^{2}\right)(1)\right]\right| \leq 154 \pi^{8} C_{1}^{3} \varepsilon r_{0}^{2} \\
\left|E_{2}-\bar{E}_{2}+2 r_{0} \psi_{0} \bar{f}_{1}^{2} \mathcal{S}_{\bar{r}_{0}}\left(t^{2}-t\right)(1)\right| \leq 84 \pi^{8} C_{1}^{3} \varepsilon r_{0}^{2} \\
\left|E_{3}-\bar{E}_{3}+4 r_{0} \psi_{1} \bar{f}_{1}\left[\bar{f}_{1} \mathcal{S}_{\bar{r}_{0}}(t)(1)-\bar{f}_{0} \mathcal{S}_{\bar{r}_{0}}\left(t^{2}\right)(1)\right]\right| \leq 200 \pi^{8} C_{1}^{3} \varepsilon r_{0}^{2}
\end{gathered}
$$

where the constant $C_{1}$ is defined $^{9}$ in Appendix $\mathrm{B}$, as well as three other constants $c_{8}, c_{9}, c_{10}$, and where, recalling Remark $2, \pi^{8}$ is used as an upper bound for $\max \left(1, r_{0}^{p-2}\right)$ with $^{10} 2 \leq p \leq 10$. Since $\bar{r}_{0}<\pi$, we may divide by $\bar{f}_{1}^{3}>0$ the resulting Maclaurin approximation estimate for $f_{1}^{3} \mathcal{C}\left(m_{0}, V_{0}\right)(\xi, \nu)$ and, using the general inequalities:

$$
\begin{aligned}
\left|\cos ^{2} \vartheta-\cos ^{2}(\vartheta+\varphi)\right| & \leq \sin ^{2} \vartheta+2 \cos ^{2} \vartheta \sin ^{2} \varphi \\
2|\cos \vartheta \sin \vartheta \sin \varphi| & \leq \sin ^{2} \vartheta+\cos ^{2} \vartheta \sin ^{2} \varphi,
\end{aligned}
$$

we obtain the estimate of Corollary 1.

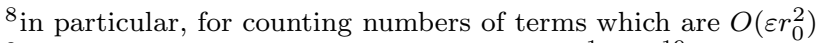

${ }^{9}$ using, in particular, the bounds $\sqrt{\kappa} \leq 1+\frac{1}{2 \pi^{2}}<\frac{19}{18}$ and $\kappa \leq 1+\frac{1}{\pi^{2}}<\frac{10}{9}$

${ }^{10}$ for instance, $p=2$ (resp. $p=10$ ) for $\left(S_{1}^{(0,1)}\right)^{2} S_{3}^{(2,0)}$ (resp. $\left(S_{3}^{(0,1)}\right)^{2} S_{3}^{(2,0)}$ ) in the first term of $E_{1}$
} 
Quick digression on the convexity of NoConj. The reader may wish to skip the rest of this section, devoted to a quick digression from our main topic. Indeed, let us pause and provide a uniform convexity estimate on the tangential domains

$$
\mathrm{NoConj}_{m}=\left\{W \in T_{m} S,(m, W) \in \mathrm{NoConj},\right.
$$

obtained in terms of $|K-1|_{C^{2}(S)}$ as a direct consequence of Lemma 1, and stated as follows:

Corollary 2. Let $S$ be a surface as above with: $\min _{S} K=1$. There exists universal positive constants $\beta, \gamma, C$, with $\beta \leq \frac{1}{\pi^{2}}$ and $\gamma \leq C$, such that, if $\mid K-$ $\left.1\right|_{C^{2}(S)} \leq \beta$, for each $m_{0} \in S$ and $V_{0} \in \partial \mathrm{NoConj}_{m_{0}}$, the curvature of the boundary curve $\partial \mathrm{NoConj}_{m_{0}}$ at $V_{0}$ is pinched between $\gamma$ and $C$.

Qualitative proofs of the uniform convexity of NoConj are given in $[5,10]$ for $C^{4}$ perturbations of the standard $n$-sphere. Let us further note that, combining Corollary 2 with Theorem 1, one can readily show that NoCut is convex for small enough $\beta$ by arguing as in [10], here just with a linear path $t \in[0,1] \rightarrow V_{t}=t V_{1}+(1-t) V_{0}$ in $T_{m_{0}} S$, with $V_{0}$ and $V_{1}$ in NoCut $m_{0}$.

Proof. Fix $\left(m_{0}, V_{0}\right)$ as stated and take a Fermi chart $x$ along $V_{0}$, sticking to the above notations. From the vanishing of $f_{1}\left(0, v_{0}, t\right)$ at $t=1$ combined with its positivity for $t \in(0,1)$ and the uniqueness of the solution of the initial (here final, rather) value problem [20], we infer that $\dot{f}_{1}\left(0, v_{0}, 1\right)<0$ hence also $D_{2} f_{1}\left(0, v_{0}, 1\right)<0$, since $f_{1}\left(0, v_{0}, t\right)=f_{1}\left((0,0),\left(0, r_{0}\right), t\right) \equiv t f_{1}\left((0,0),\left(0, t r_{0}\right), 1\right)$. Therefore, near $V_{0}$, the curve $\partial \operatorname{NoConj}_{m_{0}}$ admits the equation $v^{2}=h\left(v^{1}\right)$ with the function $h$ implicitly given by:

$$
f_{1}\left((0,0),\left(v^{1}, h\left(v^{1}\right)\right), 1\right)=0, \text { and } h(0)=r_{0} .
$$

Now, classically [3], the curvature $\mathbf{k}$ of $\partial \operatorname{NoConj}_{m_{0}}$ at $V_{0}$ is equal to:

$$
\mathbf{k}=\frac{-h^{\prime \prime}(0)}{\left(1+h^{\prime}(0)^{2}\right)^{3 / 2}} \equiv-\frac{D_{11} f_{1}\left(D_{2} f_{1}\right)^{2}-2 D_{12} f_{1}\left(D_{1} f_{1}\right)\left(D_{2} f_{1}\right)+D_{22} f_{1}\left(D_{1} f_{1}\right)^{2}}{\left(\left(D_{1} f_{1}\right)^{2}+\left(D_{2} f_{1}\right)^{2}\right)^{3 / 2}}
$$

Considering this formula, and since with $\varepsilon=0$ we would have $r_{0}=\pi, f_{1}=\bar{f}_{1}$ and $\mathbf{k}=\frac{1}{\pi^{2}}$, the timeliness of Lemma 1 for our purpose is fully conceivable. For an effective proof, we first observe that, by Sturm theorem [4], $r_{0}$ is pinched between $\pi / \sqrt{1+\varepsilon}$ and $\pi$; in particular, we have:

$$
\pi\left(1-\frac{\varepsilon}{2}\right) \leq \bar{r}_{0} \leq \pi\left(1+\frac{\varepsilon}{2}\right)
$$

At $(x, v, t)=\left(0, v_{0}, t\right)$, Lemma 1 and the formulas of Section 2.2 imply:

$$
\begin{aligned}
& D_{2} f_{1} \leq \frac{1}{r_{0}}\left(\cos \bar{r}_{0}-\frac{\sin \bar{r}_{0}}{\bar{r}_{0}}\right)+B_{211} \varepsilon r_{0} \\
& D_{11} f_{1} \leq \frac{1}{r_{0}^{2}}\left(\cos \bar{r}_{0}-\frac{\sin \bar{r}_{0}}{\bar{r}_{0}}\right)+B_{221} \varepsilon
\end{aligned}
$$


which, combined with the pinching of $r_{0}$ and standard bounds on the cosine and sine, yields:

$$
\begin{aligned}
& D_{2} f_{1} \leq \frac{1}{r_{0}}\left(-1+\varepsilon\left(\frac{1}{2}+B_{211} \pi^{2}\right)+\frac{\varepsilon^{2} \pi^{2}}{8}\right), \\
& D_{11} f_{1} \leq \frac{1}{r_{0}^{2}}\left(-1+\varepsilon\left(\frac{1}{2}+B_{221} \pi^{2}\right)+\frac{\varepsilon^{2} \pi^{2}}{8}\right) .
\end{aligned}
$$

So $D_{2} f_{1} \leq-\frac{1}{2 r_{0}}$, hence in particular $\left|D f_{1}\right| \geq \frac{1}{2 r_{0}} \geq \frac{1}{2 \pi}$, and $D_{11} f_{1} \leq-\frac{1}{2 r_{0}^{2}}$, provided $\varepsilon$ is small enough. Moreover, still by Lemma 1 and Section 2.2, we have at $\left(0, v_{0}, t\right)$ :

$$
\left|D_{1} f_{1}\right| \leq B_{211} \varepsilon r_{0} \leq B_{211} \pi \varepsilon .
$$

The combination of Lemma 1 (including Remark 4) with the preceding bounds yields, on the one hand:

$$
\mathbf{k} \leq(2 \pi)^{3} 6 B_{121} B_{111}^{2}=: C
$$

on the other hand:

$$
\mathbf{k} \geq \frac{1}{2 B_{111}^{3} \sqrt{2}}\left(\frac{1}{8 r_{0}^{4}}-\varepsilon \pi B_{211} B_{121}\left(4 B_{111}+\varepsilon \pi B_{211}\right)\right)
$$

so that $\mathbf{k} \geq \gamma:=\frac{1}{32 \pi^{4} \sqrt{2} B_{111}^{3}}$ for $\varepsilon$ small enough. Altogether, this pinching of $\mathbf{k}$ holds provided we require $\varepsilon \leq \beta$ with $\beta$ the smallest among the positive roots of the quadratic equations ${ }^{11}$ :

$$
\begin{gathered}
\frac{\pi^{2}}{8} \beta^{2}+\left(\frac{1}{2}+B_{221} \pi^{2}\right) \beta-\frac{1}{2}=0, \\
\pi^{2} B_{121} B_{211}^{2} \beta^{2}+4 \pi B_{111} B_{121} B_{211} \beta-\frac{1}{16 \pi^{4}}=0 .
\end{gathered}
$$

Finally, a tedious but routine evaluation shows that we may take:

$$
\gamma=1.1 \times 10^{-7}, C=7.4 \times 10^{7}, \beta=4.5 \times 10^{-10},
$$

in the statement of Corollary 2.

4. $c$-curvature almost-positivity near conjugacy. In this section, we prove Theorem 2 at $\left(m_{0}, V_{0}\right) \in$ NoConj and $(\xi, \nu)$ unit vectors of $T_{m_{0}} S$ in case the point $\exp _{m_{0}}\left(V_{0}\right)$ is close to the first conjugate point $m_{0}^{*}$ of $m_{0}$ along the geodesic $t \in \mathbb{R}^{+} \rightarrow$ $\exp _{m_{0}}\left(t V_{0}\right) \in S$. Specifically, setting $l_{0}$ for the length of that geodesic curve from $m_{0}$ up to $m_{0}^{*}$, we establish the following proposition:

PROPOSITION 1. There exists a triple of small (strictly) positive real numbers $\left(\eta_{1}, \delta_{1}, \varsigma_{1}\right)$ such that $\mathcal{C}\left(m_{0}, V_{0}\right)(\xi, \nu)$ satisfies the lower bound (2) with $\varsigma=\varsigma_{1}$, provided $\varepsilon=|K-1|_{C^{2}(S)} \leq \eta_{1}$ and $\left(1-\delta_{1}\right) l_{0} \leq\left|V_{0}\right|<l_{0}$.

\footnotetext{
${ }^{11}$ which turns out to be that of the second equation
} 
Proof. Sticking to previous notations and recalling (12), we infer from the pinching of $\left|V_{0}\right|$ the following ones (dropping the subscript of $\delta_{1}$ ):

$$
\left(1-\frac{\varepsilon}{2}-\delta\right) \pi \leq r_{0} \leq \pi \text { and }\left(1-\frac{\varepsilon}{2}-\delta\right) \pi \leq \bar{r}_{0}<\left(1+\frac{\varepsilon}{2}\right) \pi .
$$

We will assume:

$$
r_{0} \in\left(\frac{5 \pi}{6}, \pi\right) \text { and } \bar{r}_{0} \in\left(\frac{5 \pi}{6}, \frac{7 \pi}{6}\right)
$$

with no loss of generality (it holds under the smallness condition posed below ${ }^{12}$ on $\varepsilon$ and $\delta$, see (50) of Appendix B). Combining (13) with Remark 3, the formulas of Section 2.2 and the first line of conclusion of Lemma 1, we derive the following set of inequalities:

$$
\begin{gathered}
-1-\varepsilon \pi^{2} B_{200} \leq f_{0} \leq-1+\varepsilon \pi^{2} B_{200}+\left(\frac{\varepsilon}{2}+\delta\right)^{2} \frac{\pi^{2}}{2} \\
0<f_{1} \leq \frac{\sin r_{0}}{r_{0}} \leq \frac{\frac{\varepsilon}{2}+\delta}{1-\left(\frac{\varepsilon}{2}+\delta\right)} \\
\left|f_{0}^{\prime}\right| \leq\left(\frac{\varepsilon}{2}+\delta\right)\left(1+\frac{\varepsilon}{2}\right) \pi+\varepsilon \pi B_{210} .
\end{gathered}
$$

Furthermore, we derive two important lower bounds, namely:

LEMMA 2. If $\cos \varphi \neq 0$, and $\varepsilon$ and $\delta$ satisfy the relative smallness condition ${ }^{13}$ (49) (see Appendix B), the lower bound:

$$
\left|f_{1}^{\prime}\right| \geq \frac{|\cos \varphi|}{\pi}-\varepsilon \pi B_{211}-\frac{\frac{\varepsilon}{2}}{\pi\left(1-\left(\frac{\varepsilon}{2}+\delta\right)\right)}-\left(\frac{\varepsilon}{2}+\delta\right)^{2} \frac{\pi}{2}
$$

holds true, as well as the sign condition: $-f_{1}^{\prime} \cos \varphi>0$. If $|\cos \varphi| \leq \frac{1}{2}$ and $\varepsilon$ and $\delta$ are (50)-small, the following lower bound is valid instead:

$$
f_{0} f_{1}^{\prime \prime} \geq \frac{1}{8 \pi^{2}}-\varepsilon\left(B_{221}+\frac{1}{8} B_{200}\right)-\frac{1}{16}\left(\frac{\varepsilon}{2}+\delta\right)^{2}
$$

Proof. If $\cos \varphi \neq 0$, using $\left|f_{1}^{\prime}-\bar{f}_{1}^{\prime}\right| \leq \varepsilon \pi B_{211}$ combined with the lower bound:

$$
-\frac{\bar{f}_{1}^{\prime}}{\cos \varphi} \geq \frac{1}{\pi}\left(1-\frac{\frac{\varepsilon}{2}}{1-\left(\frac{\varepsilon}{2}+\delta\right)}-\frac{\pi^{2}}{2}\left(\frac{\varepsilon}{2}+\delta\right)^{2}\right)
$$

one can readily check the first part of the lemma. For the second part, we first note that $\bar{f}_{1}^{\prime \prime}$ is bounded above by the expression:

$$
\frac{1}{r_{0}^{2}}\left[-1+\frac{\frac{\varepsilon}{2}}{1-\left(\frac{\varepsilon}{2}+\delta\right)}+\frac{\pi^{2}}{2}\left(\frac{\varepsilon}{2}+\delta\right)^{2}+\cos ^{2} \varphi\left(\frac{3}{1-\left(\frac{\varepsilon}{2}+\delta\right)}+\frac{\varepsilon}{2}\left(1+\frac{\varepsilon}{2}\right) \pi^{2}\right)\right] .
$$

\footnotetext{
12 we will say, for short, that they are (50)-small

13 to be used only in Section 4.2 below, with $|\cos \varphi|$ bounded away from 0 by a (small) universal constant i.e. with $|\cos \varphi|$ replaced by that constant
} 
If $|\cos \varphi| \leq \frac{1}{2}$, it implies $\bar{f}_{1}^{\prime \prime} \leq-\frac{1}{8 \pi^{2}}$ provided $\varepsilon$ and $\delta$ are taken (50)-small. By Lemma 1 , the inequality

$$
f_{1}^{\prime \prime} \leq-\frac{1}{8 \pi^{2}}+\varepsilon B_{221}
$$

follows. Combined with (15), it yields the second part of the lemma.

In order to investigate the sign of the $c$-curvature expression (6), we will have to recast this expression in appropriate forms, namely, either:

$$
\begin{aligned}
\mathcal{C}\left(m_{0}, V_{0}\right)(\xi, \nu)= & -\sin ^{2} \vartheta\left(\frac{f_{0}^{\prime \prime}}{f_{1}}-\frac{f_{0} f_{1}^{\prime \prime}}{f_{1}^{2}}-\frac{2 f_{0}^{\prime} f_{1}^{\prime}}{f_{1}^{2}}\right) \\
& -\frac{2 f_{0}}{f_{1}}\left(\frac{f_{1}^{\prime}}{f_{1}} \sin \vartheta+\frac{\sin \varphi \cos \vartheta}{r_{0}}\right)^{2} \\
& +\frac{2}{r_{0}^{2}}\left(1-\frac{f_{0}}{f_{1}}\right)\left(2 \cos \vartheta \cos \varphi \sin \vartheta \sin \varphi-\sin ^{2} \vartheta \sin ^{2} \varphi\right) \\
& +\frac{2}{r_{0}^{2}} \cos ^{2} \vartheta \sin ^{2} \varphi+\frac{4}{r_{0}} \cos \vartheta \sin \vartheta \sin \varphi \frac{f_{0}^{\prime}}{f_{1}}
\end{aligned}
$$

or:

$$
\begin{aligned}
\mathcal{C}\left(m_{0}, V_{0}\right)(\xi, \nu)= & -\sin ^{2} \vartheta\left(\frac{f_{0}^{\prime \prime}}{f_{1}}-\frac{f_{0} f_{1}^{\prime \prime}}{f_{1}^{2}}-\frac{2 f_{0}^{\prime} f_{1}^{\prime}}{f_{1}^{2}}+\frac{2 f_{0}\left(f_{1}^{\prime}\right)^{2}}{f_{1}^{3}}+\frac{2}{r_{0}^{2}}\left(1-\frac{f_{0}}{f_{1}}\right)\right) \\
& +\frac{2}{r_{0}^{2}}\left(1-\frac{f_{0}}{f_{1}}\right)(\cos \varphi \sin \vartheta+\cos \vartheta \sin \varphi)^{2} \\
& +\frac{4}{r_{0}} \cos \vartheta \sin \vartheta \sin \varphi\left(\frac{f_{0}^{\prime}}{f_{1}}-\frac{f_{0} f_{1}^{\prime}}{f_{1}^{2}}\right),
\end{aligned}
$$

We will also have to distinguish cases, depending on the size of $|\cos \varphi|$, then on the relative size of further arising quantities. In each case, relying on Lemma 2 and treating $f_{1}$ as a small parameter in intermediate steps thanks to (16), we will be able to find a leading term blowing up positively as $\varepsilon$ and $\delta$ go to zero and argue with it.

We are now ready to continue the proof of Proposition 1 and start out for a case by case discussion of the sign of the $c$-curvature.

\subsection{Case $|\cos \varphi|$ small enough.}

4.1.1. Subcase $\left|\sin \vartheta \frac{f_{1}^{\prime}}{f_{1}}\right| \leq \frac{|\sin \varphi \cos \vartheta|}{2 r_{0}}$. In this subcase, the assumption $|\cos \varphi| \leq \frac{1}{2}$ will suffice. We note the estimate:

$$
\left(\frac{f_{1}^{\prime}}{f_{1}} \sin \vartheta+\frac{\sin \varphi \cos \vartheta}{r_{0}}\right)^{2} \geq \frac{\sin ^{2} \varphi \cos ^{2} \vartheta}{4 r_{0}^{4}}
$$

and use it to derive from (18) the inequality:

$$
\begin{aligned}
\mathcal{C}\left(m_{0}, V_{0}\right)(\xi, \nu) & \geq \frac{\sin ^{2} \vartheta}{f_{1}^{2}}\left[f_{0} f_{1}^{\prime \prime}+2 f_{0}^{\prime} f_{1}^{\prime}-f_{1}\left(f_{0}^{\prime \prime}+\frac{2}{r_{0}^{2}}\left(f_{1}-f_{0}\right) \sin ^{2} \varphi\right)\right] \\
& -\frac{2 f_{0}}{f_{1}} \frac{\sin ^{2} \varphi \cos ^{2} \vartheta}{4 r_{0}^{2}} \\
& -\frac{4}{f_{1}}|\cos \vartheta \sin \vartheta \sin \varphi|\left(\frac{f_{1}-f_{0}}{r_{0}^{2}}+\frac{\left|f_{0}^{\prime}\right|}{r_{0}}\right) .
\end{aligned}
$$


The right-hand side will be handled relying on the second part of Lemma 2 combined with the pinching (14) of $r_{0}$ and previous estimates on the various $D^{k} f_{a}$ terms which arise apart from $f_{0} f_{1}^{\prime \prime}$. Doing so, we can establish for $\mathcal{C}\left(m_{0}, V_{0}\right)(\xi, \nu)$ the lower bound:

$$
\begin{aligned}
\mathcal{C}\left(m_{0}, V_{0}\right)(\xi, \nu) & \geq \frac{\sin ^{2} \vartheta}{f_{1}^{2}}\left(\frac{1}{12 \pi^{2}}-R_{1}(\varepsilon, \delta)\right)+\frac{\sin ^{2} \varphi \cos ^{2} \vartheta}{8 \pi^{2} f_{1}} \\
& +\frac{\sin ^{2} \vartheta}{24 \pi^{2} f_{1}^{2}}+\frac{\sin ^{2} \varphi \cos ^{2} \vartheta}{8 \pi^{2} f_{1}}-4\left(1+\frac{5}{\pi^{2}}\right) \frac{|\cos \vartheta \sin \vartheta \sin \varphi|}{f_{1}}
\end{aligned}
$$

provided $\varepsilon$ and $\delta$ are (51)-small, where $R_{1}(\varepsilon, \delta)$ stands for the rational function of $(\varepsilon, \delta)$ vanishing at $(0,0)$ given by the right-hand side of the smallness condition (51). We claim that the second line of the right-hand side of $(20)$ is non-negative for small enough $\varepsilon$ and $\delta$. Indeed, from the identity $a^{2}+b^{2} \geq 2|a b|$ used with $a=\frac{\sin \vartheta}{2 \pi \sqrt{6} f_{1}}$ and $b=\frac{\sin \varphi \cos \vartheta}{2 \pi \sqrt{2 f_{1}}}$, we infer that this line is bounded below by:

$$
\frac{|\cos \vartheta \sin \vartheta \sin \varphi|}{f_{1} \sqrt{f_{1}}}\left(\frac{1}{4 \sqrt{3} \pi^{2}}-4\left(1+\frac{5}{\pi^{2}}\right) \sqrt{f_{1}}\right),
$$

and the claim follows by taking $\varepsilon$ and $\delta$ (52)-small. Eventually, for $\varepsilon$ and $\delta$ (51)(52)small, we obtain:

$$
\mathcal{C}\left(m_{0}, V_{0}\right)(\xi, \nu) \geq \frac{\sin ^{2} \vartheta}{24 \pi^{2} f_{1}^{2}}+\frac{\sin ^{2} \varphi \cos ^{2} \vartheta}{8 \pi^{2} f_{1}} .
$$

Combining this lower bound with (16)(52) and the useful, easily established ${ }^{14}$, inequality:

$$
\sin ^{2} \vartheta+\cos ^{2} \vartheta \sin ^{2} \varphi \geq \frac{1}{4 \pi^{2}} \mathcal{A}_{2}\left(m_{0}, V_{0}, \xi, \nu\right)
$$

we get $(2)$ at $\left(m_{0}, V_{0}, \xi, \nu\right)$ with $\varsigma=18$.

4.1.2. Subcase $\left|\sin \vartheta \frac{f_{1}^{\prime}}{f_{1}}\right|>\frac{|\sin \varphi \cos \vartheta|}{2 r_{0}}$. The second line of the right-hand side of (18) is non-negative due to $(15)(16)$. So we may write:

$$
\begin{aligned}
\mathcal{C}\left(m_{0}, V_{0}\right)(\xi, \nu) & \geq \frac{\sin ^{2} \vartheta}{f_{1}^{2}}\left[f_{0} f_{1}^{\prime \prime}+2 f_{0}^{\prime} f_{1}^{\prime}-f_{1}\left(f_{0}^{\prime \prime}+\frac{2}{r_{0}^{2}}\left(f_{1}-f_{0}\right) \sin ^{2} \varphi\right)\right] \\
& -\frac{4}{f_{1}}|\cos \vartheta \sin \vartheta \sin \varphi|\left(\frac{f_{1}-f_{0}}{r_{0}^{2}}|\cos \varphi|+\frac{\left|f_{0}^{\prime}\right|}{r_{0}}\right),
\end{aligned}
$$

hence also:

$$
\begin{aligned}
\mathcal{C}\left(m_{0}, V_{0}\right)(\xi, \nu) & \geq \frac{\sin ^{2} \vartheta}{f_{1}^{2}}\left[f_{0} f_{1}^{\prime \prime}+2 f_{0}^{\prime} f_{1}^{\prime}-f_{1}\left(f_{0}^{\prime \prime}+\frac{2}{r_{0}^{2}}\left(f_{1}-f_{0}\right) \sin ^{2} \varphi\right)\right] \\
& -\frac{8 \sin ^{2} \vartheta}{f_{1}^{2}}\left|f_{1}^{\prime}\right|\left(\frac{f_{1}-f_{0}}{r_{0}}|\cos \varphi|+\left|f_{0}^{\prime}\right|\right)
\end{aligned}
$$

\footnotetext{
${ }^{14}$ hint: use Remark 2
} 
by applying our subcase assumption. If $|\cos \varphi| \leq \frac{1}{2}$, repeating the above argument, we see that the first line of the preceding right-hand side is larger than $\frac{\sin ^{2} \vartheta}{f_{1}^{2}}\left(\frac{1}{8 \pi^{2}}-R_{1}(\varepsilon, \delta)\right)$, while the second line is bounded below by:

$$
-8 B_{111} \frac{\sin ^{2} \vartheta}{f_{1}^{2}}\left[\left(\frac{\varepsilon}{2}+\delta\right)\left(1+\frac{\varepsilon}{2}\right) \pi+\varepsilon \pi B_{210}+\frac{1+\varepsilon \pi^{2} B_{200}+\frac{\frac{\varepsilon}{2}+\delta}{1-\left(\frac{\varepsilon}{2}+\delta\right)}}{\pi\left(1-\frac{\varepsilon}{2}-\delta\right)}|\cos \varphi|\right]
$$

as shown by combining Lemma 1 with (13)(15)(16)(17). Altogether, we may write:

$$
\begin{aligned}
\mathcal{C}\left(m_{0}, V_{0}\right)(\xi, \nu) & \geq \frac{\sin ^{2} \vartheta}{f_{1}^{2}}\left(\frac{1}{12 \pi^{2}}-R_{2}(\varepsilon, \delta)\right) \\
& +\frac{\sin ^{2} \vartheta}{f_{1}^{2}}\left(\frac{1}{24 \pi^{2}}-8 B_{111} \frac{1+\varepsilon \pi^{2} B_{200}+\frac{\frac{\varepsilon}{2}+\delta}{1-\left(\frac{\varepsilon}{2}+\delta\right)}}{\pi\left(1-\frac{\varepsilon}{2}-\delta\right)}|\cos \varphi|\right)
\end{aligned}
$$

with $R_{2}(\varepsilon, \delta)$ given by the right-hand side of (53). We get from (52):

$$
\frac{1+\varepsilon \pi^{2} B_{200}+\frac{\frac{\varepsilon}{2}+\delta}{1-\left(\frac{\varepsilon}{2}+\delta\right)}}{\left(1-\frac{\varepsilon}{2}-\delta\right)} \leq \frac{768\left(\pi^{2}+5\right)^{2}+2 \pi^{2}+1}{768\left(\pi^{2}+5\right)^{2}-1}<1.00013
$$

besides, we have: $\pi B_{111}=5+\pi \sqrt{2}+3 \pi^{2} \simeq 39,05<40$. So the smallness conditions:

$$
|\cos \varphi| \leq \frac{1}{7704}
$$

and (53) imply that $\mathcal{C}\left(m_{0}, V_{0}\right)(\xi, \nu) \geq \frac{\sin ^{2} \vartheta}{24 \pi^{2} f_{1}^{2}}$. In our present subcase, the latter inequality yields:

$$
\mathcal{C}\left(m_{0}, V_{0}\right)(\xi, \nu) \geq \frac{\sin ^{2} \vartheta}{48 \pi^{2} f_{1}^{2}}+\frac{\cos ^{2} \vartheta \sin ^{2} \varphi}{192 \pi^{2} r_{0}^{2} f_{1}^{\prime 2}} .
$$

On the one hand, from (16) combined with (52), we get $48 \pi^{2} f_{1}^{2} \leq 2 \times 10^{-8}$. On the other hand, combining Lemma 1 with (13) and (22), we have: $r_{0}\left|f_{1}^{\prime}\right| \leq \varepsilon \pi^{2} B_{211}+\frac{1}{3852}$. So we can arrange to have $192 \pi^{2} r_{0}^{2} f_{1}^{\prime 2} \leq 1$ by taking $\varepsilon(54)$-small. Altogether, we may write

$$
\mathcal{C}\left(m_{0}, V_{0}\right)(\xi, \nu) \geq \sin ^{2} \vartheta+\cos ^{2} \vartheta \sin ^{2} \varphi
$$

and, from (21), conclude that (2) holds at $\left(m_{0}, V_{0}, \xi, \nu\right)$, indeed, with $\varsigma=\frac{1}{4 \pi^{2}}$.

4.2. Case $|\cos \varphi|>\frac{1}{7704}$. In this case, the first part of Lemma 2 implies:

$$
\left|f_{1}^{\prime}\right| \geq \frac{1}{15408 \pi} \text { with }-f_{1}^{\prime} \cos \varphi>0,
$$

provided $\varepsilon$ and $\delta$ are (55)-small. Furthermore, if the latter are (50)(51)-small, we infer from (15) the pinching:

$$
\frac{1}{2} \leq-f_{0} \leq \frac{3}{2} .
$$

which will be used repeatedly. 
4.2.1. Subcase $\cos \vartheta \cos \varphi \sin \vartheta \sin \varphi \leq 0$. Working with the expression (19) of $\mathcal{C}\left(m_{0}, V_{0}\right)(\xi, \nu)$, the second line of which is non-negative, and combining (23) with (24), (14) and Lemma 1, we get the inequality:

$$
\begin{aligned}
\mathcal{C}\left(m_{0}, V_{0}\right)(\xi, \nu) & \geq \frac{\sin ^{2} \vartheta}{f_{1}^{3}}\left(\frac{1}{15408^{2} \pi^{2}}-f_{1}\left(B_{120}+\frac{3}{2} B_{121}+2 B_{110} B_{111}+\frac{36}{5 \pi^{2}}\right)\right) \\
& +\frac{2}{r_{0}^{2}}\left(1-\frac{f_{0}}{f_{1}}\right)(\cos \varphi \sin \vartheta+\cos \vartheta \sin \varphi)^{2} \\
& +\frac{2}{r_{0} f_{1}^{2}}|\cos \vartheta \sin \vartheta \sin \varphi|\left(\frac{1}{15408 \pi}-2 f_{1} B_{110}\right) .
\end{aligned}
$$

Recalling (16) (24) and assuming that $\varepsilon$ and $\delta$ are (56)-small, we infer the lower bound:

$$
\begin{aligned}
\mathcal{C}\left(m_{0}, V_{0}\right)(\xi, \nu) & \geq \frac{\sin ^{2} \vartheta}{2 \pi^{2} 15408^{2} f_{1}^{3}}+\frac{2}{r_{0}^{2}}\left(1+\frac{1}{2 f_{1}}\right) \cos ^{2} \vartheta \sin ^{2} \varphi \\
& +\frac{1}{15408 \pi r_{0} f_{1}^{2}}|\cos \vartheta \sin \vartheta \sin \varphi|\left(1-\frac{4}{r_{0}} 15408 \pi f_{1}\left(f_{1}-f_{0}\right)\right)
\end{aligned}
$$

the second line of the right-hand side of which is non-negative, as checked by combining Remark 3 with (13) (16) (24) and (52). Using (13)(16)(52) to treat its first line, we obtain the inequality

$$
\mathcal{C}\left(m_{0}, V_{0}\right)(\xi, \nu) \geq 17205\left(\sin ^{2} \vartheta+\cos ^{2} \vartheta \sin ^{2} \varphi\right)
$$

which, recalling $(21)$, implies $(2)$ at $\left(m_{0}, V_{0}, \xi, \nu\right)$ with $\varsigma=435$.

4.2.2. Subcase $\cos \vartheta \cos \varphi \sin \vartheta \sin \varphi>0$. Here, since $-f_{1}^{\prime} \cos \varphi>0$, we know that the expressions $\sin \vartheta \frac{f_{1}^{\prime}}{f_{1}}$ and $\frac{\sin \varphi \cos \vartheta}{r_{0}}$ have opposite signs.

Case $\left|\sin \vartheta \frac{f_{1}^{\prime}}{f_{1}}\right| \leq \frac{4|\sin \varphi \cos \vartheta|}{5 r_{0}}$ or $\left|\sin \vartheta \frac{f_{1}^{\prime}}{f_{1}}\right| \geq \frac{5|\sin \varphi \cos \vartheta|}{4 r_{0}}$. If $a$ and $b$ are two real numbers such that: $a b<0$ and $|a| \leq \frac{4}{5}|b|$ or $|b| \leq \frac{4}{5}|a|$, one can readily verify that they satisfy: $(a+b)^{2} \geq \frac{1}{50}\left(a^{2}+b^{2}\right)$. Using the expression $(18)$ of $\mathcal{C}\left(m_{0}, V_{0}\right)(\xi, \nu)$, we apply the preceding estimate with $a=\sin \vartheta \frac{f_{1}^{\prime}}{f_{1}}, b=\frac{\sin \varphi \cos \vartheta}{r_{0}}$, and find the $c$ curvature bounded below by:

$$
\begin{aligned}
& \sin ^{2} \vartheta\left(\frac{-f_{0}\left(f_{1}^{\prime}\right)^{2}}{25 f_{1}^{3}}-\frac{f_{0}^{\prime \prime}}{f_{1}}+\frac{f_{0} f_{1}^{\prime \prime}+2 f_{0}^{\prime} f_{1}^{\prime}}{f_{1}^{2}}-\frac{2}{r_{0}^{2}} \sin ^{2} \varphi\left(1-\frac{f_{0}}{f_{1}}\right)\right) \\
& +\frac{4|\cos \vartheta \sin \vartheta \sin \varphi|}{f_{1}}\left(-\frac{f_{0}}{7704 r_{0}^{2}}-\frac{\left|f_{0}^{\prime}\right|}{r_{0}}\right)-\frac{f_{0}}{25 f_{1} r_{0}^{2}} \cos ^{2} \vartheta \sin ^{2} \varphi,
\end{aligned}
$$

hence also, combining Lemma 1 with $(14)(23)(24)$ and (17), by:

$$
\begin{gathered}
\frac{\sin ^{2} \vartheta}{f_{1}^{3}}\left(\frac{1}{50 \pi^{2} 15408^{2}}-f_{1}\left(B_{120}+\frac{3}{2} B_{121}+2 B_{110} B_{111}+\frac{36}{5 \pi^{2}}\right)\right) \\
+\frac{4|\cos \vartheta \sin \vartheta \sin \varphi|}{f_{1}}\left(\frac{1}{15408 \pi^{2}}-\frac{6}{5}\left(\left(\frac{\varepsilon}{2}+\delta\right)\left(1+\frac{\varepsilon}{2}\right)+\varepsilon B_{210}\right)\right)+\frac{1}{50 \pi^{2} f_{1}} \cos ^{2} \vartheta \sin ^{2} \varphi .
\end{gathered}
$$


Recalling (16), we infer that:

$$
\mathcal{C}\left(m_{0}, V_{0}\right)(\xi, \nu) \geq \frac{\sin ^{2} \vartheta}{100 \pi^{2} 15408^{2} f_{1}^{3}}+\frac{1}{50 \pi^{2} f_{1}} \cos ^{2} \vartheta \sin ^{2} \varphi,
$$

provided $\varepsilon$ and $\delta$ are (57)-small. Recalling (16)(52) and (21), it yields (2) with $\varsigma=8.7$ at $\left(m_{0}, V_{0}, \xi, \nu\right)$.

Case $\frac{4|\sin \varphi \cos \vartheta|}{5 r_{0}}<\left|\sin \vartheta \frac{f_{1}^{\prime}}{f_{1}}\right|<\frac{5|\sin \varphi \cos \vartheta|}{4 r_{0}}$. This case is more difficult because we cannot use the square occuring in the second line of (18) any more; all we can do now from (18) is write:

$$
\begin{aligned}
\mathcal{C}\left(m_{0}, V_{0}\right)(\xi, \nu) & \geq \sin ^{2} \vartheta\left(-\frac{f_{0}^{\prime \prime}}{f_{1}}+\frac{f_{0} f_{1}^{\prime \prime}+2 f_{0}^{\prime} f_{1}^{\prime}}{f_{1}^{2}}-\frac{2}{r_{0}^{2}} \sin ^{2} \varphi\left(1-\frac{f_{0}}{f_{1}}\right)\right) \\
& +\frac{4}{r_{0}^{2}}|\sin \varphi \cos \vartheta||\cos \varphi \sin \vartheta|\left(1-\frac{f_{0}}{f_{1}}\right) \\
& -\frac{4}{r_{0}}|\sin \varphi \cos \vartheta||\sin \vartheta| \frac{\left|f_{0}^{\prime}\right|}{f_{1}}
\end{aligned}
$$

and, from our present assumption, infer for $\mathcal{C}\left(m_{0}, V_{0}\right)(\xi, \nu)$ the lower bound:

$$
\begin{aligned}
& \sin ^{2} \vartheta\left(-\frac{f_{0}^{\prime \prime}}{f_{1}}+\frac{f_{0} f_{1}^{\prime \prime}+2 f_{0}^{\prime} f_{1}^{\prime}}{f_{1}^{2}}-\frac{2}{r_{0}^{2}} \sin ^{2} \varphi\left(1-\frac{f_{0}}{f_{1}}\right)\right) \\
& -\frac{16 f_{0}}{5 r_{0} f_{1}^{2}} \sin ^{2} \vartheta\left|f_{1}^{\prime} \cos \varphi\right|-5 \sin ^{2} \vartheta \frac{\left|f_{1}^{\prime} f_{0}^{\prime}\right|}{f_{1}^{2}} .
\end{aligned}
$$

We will factorize $\frac{\sin ^{2} \vartheta}{f_{1}^{2}}$ as leading blowing up term in this expression and seek a positive coefficient for it. Doing so, we focus on the terms:

$$
-\frac{f_{0} \sin ^{2} \vartheta}{f_{1}^{2}}\left(-f_{1}^{\prime \prime}+\frac{16}{5 r_{0}}\left|f_{1}^{\prime} \cos \varphi\right|\right)
$$

thus carefully investigate the sign of the latter parenthesis. Using Lemma 1, we find it bounded below by:

$$
\left(-\bar{f}_{1}^{\prime \prime}+\frac{16}{5 r_{0}}\left|\bar{f}_{1}^{\prime} \cos \varphi\right|\right)-\varepsilon\left(B_{221}+\frac{16}{5} B_{211}\right) .
$$

Now, a direct calculation of $\left(-\bar{f}_{1}^{\prime \prime}+\frac{16}{5 r_{0}}\left|\bar{f}_{1}^{\prime} \cos \varphi\right|\right)$, using the expressions of $\bar{f}_{1}^{\prime}$ and $\bar{f}_{1}^{\prime \prime}$ given in Section 2.2, shows that it is equal to:

$$
\frac{1}{r_{0}^{2}}\left[\left|\cos \bar{r}_{0}\right|\left(1+\frac{1}{5} \cos ^{2} \varphi\right)+\frac{\sin \bar{r}_{0}}{\bar{r}_{0}}\left(1+\left(\bar{r}_{0}^{2}+\frac{1}{5}\right) \cos ^{2} \varphi\right)\right]
$$

recalling (13), we see that it will meet the required positivity. Back to the lower bound (25), rewritten as $\mathcal{C}\left(m_{0}, V_{0}\right)(\xi, \nu) \geq \frac{\sin ^{2} \vartheta}{f_{1}^{2}} E$ with $E$ equal to:

$$
\left(-f_{0}\right)\left(-f_{1}^{\prime \prime}+\frac{16}{5 r_{0}}\left|f_{1}^{\prime} \cos \varphi\right|\right)-7\left|f_{0}^{\prime} f_{1}^{\prime}\right|-f_{1}\left(\left|f_{0}^{\prime \prime}\right|+\frac{2 \sin ^{2} \varphi}{r_{0}^{2}}\left(f_{1}-f_{0}\right)\right),
$$


the preceding argument, combined with Lemma 1, Remark 3 and (13) (14) (16) (17) (24), implies that $\mathcal{C}\left(m_{0}, V_{0}\right)(\xi, \nu) \geq \frac{\sqrt{3} \sin ^{2} \vartheta}{8 \pi^{2} f_{1}^{2}}$ provided $\varepsilon$ and $\delta$ are (58)-small. In the present subcase, the latter inequality implies:

$$
\mathcal{C}\left(m_{0}, V_{0}\right)(\xi, \nu) \geq \frac{\sqrt{3}}{16 \pi^{2}}\left(\frac{\sin ^{2} \vartheta}{f_{1}^{2}}+\frac{16 \cos ^{2} \vartheta \sin ^{2} \varphi}{25 r_{0}^{2}\left(f_{1}^{\prime}\right)^{2}}\right) .
$$

Recalling that $r_{0}^{2}\left(f_{1}^{\prime}\right)^{2} \leq \frac{1}{192 \pi^{2}}$ due to $(54)$ and $f_{1}^{2} \leq \frac{1}{\left(16 \sqrt{3}\left(\pi^{2}+5\right)\right)^{4}}$ by $(16)(52)$, we obtain

$$
\mathcal{C}\left(m_{0}, V_{0}\right)(\xi, \nu) \geq \frac{1212 \sqrt{3}}{16 \pi^{2}}\left(\sin ^{2} \vartheta+\cos ^{2} \vartheta \sin ^{2} \varphi\right)
$$

which, combined with (21), yields (2) with $\varsigma=0.3$ at $\left(m_{0}, V_{0}, \xi, \nu\right)$.

4.3. Concluding the proof of Proposition 1. By inspection of the smallness conditions (49) through (58) which $\varepsilon$ and $\delta_{1}$ must satisfy, we find that (57) implies all others. Calculation yields the pinching:

$$
1439 \leq B_{120}+\frac{3}{2} B_{121}+2 B_{110} B_{111}+\frac{36}{5 \pi^{2}} \leq 1440
$$

the right-hand side of which provides the condition:

$$
\frac{\varepsilon}{2}+\delta_{1} \leq 2.96 \times 10^{-15}
$$

as a sufficient one for (57), hence for all, to be satisfied. It leads us to take:

$$
\eta_{1}=2.96 \times 10^{-15}, \delta_{1}=1.48 \times 10^{-15},
$$

in the statement of Proposition 1. As for $\varsigma_{1}$, we choose the smallest value among the ones found along the way, namely: $\varsigma_{1}=\frac{1}{4 \pi^{2}}$.

Finally, let us stress that the proof just completed obviously departs from that of [10] mentionned in Remark 1; in particular, in each of the above cases, the origin of the blow up rate (quadratic or cubic) chosen for the positive lower bound on the $c$ curvature can readily be traced back to the expression of $\mathcal{C}\left(m_{0}, V_{0}\right)(\xi, \nu)$ itself, relying on Lemma 2 and Lemma 1.

5. c-curvature almost-positivity near the origin. In this section, we prove Theorem 2 at $\left(m_{0}, V_{0}, \xi, \nu\right)$ when $d\left(m_{0}, \exp _{m_{0}}\left(V_{0}\right)\right)$ is small.

Proposition 2. There exists a triple of small (strictly) positive real numbers $\left(\eta_{2}, \delta_{2}, \varsigma_{2}\right)$ such that $\mathcal{C}\left(m_{0}, V_{0}\right)(\xi, \nu)$ satisfies the lower bound (2) with $\varsigma=\varsigma_{2}$, provided $\varepsilon=|K-1|_{C^{2}(S)} \leq \eta_{2}$ and $\left|V_{0}\right| \leq \delta_{2}$.

Proof. As already observed, we may take $V_{0} \neq 0$ with no loss of generality. Dropping the subscript of $\delta_{2}$, we take $\bar{r}_{0} \leq \frac{\pi}{2}$ by assuming $\varepsilon$ and $\delta$ (59)-small. We use the Maclaurin type approximation of $\frac{f_{1}^{3}}{\bar{f}_{1}^{3}} \mathcal{C}\left(m_{0}, V_{0}\right)(\xi, \nu)$ obtained in Corollary 1 and proceed to specify it further as $r_{0} \downarrow 0$. As regards its first summand, namely 
$\overline{\mathcal{C}}\left(m_{0}, V_{0}\right)(\xi, \nu)$, the expression (7) prompts us to define constants $c_{11}, \ldots, c_{14}$ as done in Appendix B. These definitions imply at once that the absolute value of:

$$
\begin{aligned}
& \overline{\mathcal{C}}\left(m_{0}, V_{0}\right)(\xi, \nu)-\frac{2 \kappa \bar{r}_{0}^{2}}{45} \sin ^{2} \vartheta \sin ^{2} \varphi-\frac{2 \kappa}{3}\left(1+\frac{2 \bar{r}_{0}^{2}}{5}\right) \sin ^{2} \vartheta \cos ^{2} \varphi \\
& -\frac{2 \kappa}{3}\left(1+\frac{2 \bar{r}_{0}^{2}}{15}\right) \cos ^{2} \vartheta \sin ^{2} \varphi-\frac{4 \kappa}{3}\left(1+\frac{\bar{r}_{0}^{2}}{5}\right) \cos \vartheta \sin \vartheta \cos \varphi \sin \varphi
\end{aligned}
$$

is bounded above by:

$$
\begin{gathered}
\kappa \bar{r}_{0}^{3}\left(c_{11} \sin ^{2} \vartheta \sin ^{2} \varphi+c_{12} \sin ^{2} \vartheta \cos ^{2} \varphi\right. \\
\left.+c_{13} \cos ^{2} \vartheta \sin ^{2} \varphi+c_{14}|\cos \vartheta \sin \vartheta \cos \varphi \sin \varphi|\right) .
\end{gathered}
$$

Let us now focus on the second summand, namely on the expression

$$
\begin{gathered}
E_{4}:=\frac{r_{0} \psi_{2} \sin ^{2} \vartheta}{\bar{f}_{1}}\left(\mathcal{S}_{\bar{r}_{0}}(t)(1)-\frac{\bar{f}_{0} \mathcal{S}_{\bar{r}_{0}}\left(t^{2}\right)(1)}{\bar{f}_{1}}\right) \\
-\frac{2 r_{0} \psi_{0} \mathcal{S}_{\bar{r}_{0}}\left(t^{2}-t\right)(1)}{\bar{f}_{1}}\left(\cos ^{2} \vartheta-\cos ^{2}(\vartheta+\varphi)\right) \\
-\frac{4 r_{0} \psi_{1} \cos \vartheta \sin \vartheta \sin \varphi}{\bar{f}_{1}}\left(\mathcal{S}_{\bar{r}_{0}}(t)(1)-\frac{\bar{f}_{0} \mathcal{S}_{\bar{r}_{0}}\left(t^{2}\right)(1)}{\bar{f}_{1}}\right)
\end{gathered}
$$

and rewrite, on the one hand:

$$
\frac{r_{0}}{\bar{f}_{1}}\left(\mathcal{S}_{\bar{r}_{0}}(t)(1)-\frac{\bar{f}_{0} \mathcal{S}_{\bar{r}_{0}}\left(t^{2}\right)(1)}{\bar{f}_{1}}\right)
$$

as: $r_{0} \mathcal{S}_{\bar{r}_{0}}\left(t-t^{2}\right)(1)+\sqrt{\kappa} r_{0}^{2}\left[A_{1}\left(\bar{r}_{0}\right) \mathcal{S}_{\bar{r}_{0}}(t)(1)-A_{2}\left(\bar{r}_{0}\right) \mathcal{S}_{\bar{r}_{0}}\left(t^{2}\right)(1)\right]$, where ${ }^{15}$ :

$$
A_{1}(\tau):=\frac{\tau-\sin \tau}{\tau \sin \tau}, A_{2}(\tau):=\frac{\tau^{2} \cos \tau-\sin ^{2} \tau}{\tau \sin ^{2} \tau}
$$

(and note that two additional constants $c_{15}, c_{16}$ are defined accordingly as in Appendix B), on the other hand:

$$
\frac{r_{0}}{\bar{f}_{1}} \mathcal{S}_{\bar{r}_{0}}\left(t^{2}-t\right)(1)=r_{0} \mathcal{S}_{\bar{r}_{0}}\left(t^{2}-t\right)(1)+\sqrt{\kappa} r_{0}^{2} A_{1}\left(\bar{r}_{0}\right) \mathcal{S}_{\bar{r}_{0}}\left(t^{2}-t\right)(1) .
$$

Furthermore, the Maclaurin expansion of $\mathcal{S}_{\bar{r}_{0}}\left(t^{2}-t\right)(1)$ prompts us to write:

$$
r_{0} \mathcal{S}_{\bar{r}_{0}}\left(t^{2}-t\right)(1)=-\frac{r_{0}}{12}+\kappa r_{0}^{3} A_{3}\left(\bar{r}_{0}\right)
$$

${ }^{15}$ so that: $A_{1}\left(\bar{r}_{0}\right)=\frac{1}{\bar{r}_{0}}\left(\frac{1}{f_{1}}-1\right), A_{2}\left(\bar{r}_{0}\right)=\frac{1}{\bar{r}_{0}}\left(\frac{\bar{f}_{0}}{f_{1}^{2}}-1\right)$ 
(defining so the auxiliary function $A_{3}$ and, accordingly, a constant $c_{17}$ as in Appendix B). Gathering terms of same order and recalling (11), we obtain that the absolute value of:

$$
E_{4}-\frac{r_{0}}{6}\left[2 \sin \vartheta \sin \varphi \sin (\vartheta-\varphi) \partial_{1} K(0)+\left(2 \sin \vartheta \cos \varphi \sin (\vartheta-\varphi)+\sin ^{2}(\vartheta-\varphi)\right) \partial_{2} K(0)\right]
$$

is bounded above by:

$$
\begin{gathered}
2 \sqrt{\kappa} \varepsilon r_{0}^{2}\left[\left(8\left(c_{15} c_{6}+c_{16} c_{7}\right)+\left(c_{6}+c_{7}\right) c_{15}\right) \sin ^{2} \vartheta\right. \\
\left.+\left(4\left(c_{15} c_{6}+c_{16} c_{7}\right)+2\left(c_{6}+c_{7}\right) c_{15}\right) \cos ^{2} \vartheta \sin ^{2} \varphi\right] \\
+2 c_{17} \kappa \varepsilon r_{0}^{3}\left(9 \sin ^{2} \vartheta+6 \cos ^{2} \vartheta \sin ^{2} \varphi\right) .
\end{gathered}
$$

Combining the latter inequality with the one derived above for the first summand $\overline{\mathcal{C}}\left(m_{0}, V_{0}\right)(\xi, \nu)$ of the expansion of $\mathcal{C}\left(m_{0}, V_{0}\right)(\xi, \nu)$ given in Corollary 1 , we infer that, if we consider the decomposition:

$$
\frac{f_{1}^{3}}{\bar{f}_{1}^{3}} \mathcal{C}\left(m_{0}, V_{0}\right)(\xi, \nu)=I+I I+I I I
$$

with

$$
\begin{gathered}
I:=\frac{\kappa}{3}\left(1+\frac{23 \bar{r}_{0}^{2}}{30}\right) \sin ^{2} \vartheta \cos ^{2} \varphi+\frac{\kappa}{3}\left(1+\frac{\bar{r}_{0}^{2}}{10}\right) \cos ^{2} \vartheta \sin ^{2} \varphi \\
-\frac{2 \kappa}{3}\left(1+\frac{2 \bar{r}_{0}^{2}}{5}\right) \sin \vartheta \cos \vartheta \sin \varphi \cos \varphi
\end{gathered}
$$

and

$$
\begin{aligned}
I I:=\frac{\kappa}{3} \sin ^{2}(\vartheta-\varphi) & +\frac{\kappa \bar{r}_{0}^{2}}{180}\left(\sin ^{2} \vartheta \cos ^{2} \varphi+\cos ^{2} \vartheta \sin ^{2} \varphi+4 \sin ^{2} \vartheta \sin ^{2} \varphi\right) \\
& +\frac{r_{0}}{6}\left[2 \sin \vartheta \sin \varphi \sin (\vartheta-\varphi) \partial_{1} K(0)\right. \\
+ & \left.\left(2 \sin \vartheta \cos \varphi \sin (\vartheta-\varphi)+\sin ^{2}(\vartheta-\varphi)\right) \partial_{2} K(0)\right]
\end{aligned}
$$

and $I I I$ so defined, then the quantity:

$$
\left|I I I-\frac{\kappa \bar{r}_{0}^{2}}{180}\left(\sin ^{2} \vartheta \cos ^{2} \varphi+\cos ^{2} \vartheta \sin ^{2} \varphi+4 \sin ^{2} \vartheta \sin ^{2} \varphi\right)\right|
$$

is altogether bounded above by:

$$
\begin{gathered}
\varepsilon r_{0}^{2} \sin ^{2} \vartheta\left(\frac{338 C_{1}^{3} \pi^{8}}{\bar{f}_{1}^{3}}+2 \sqrt{\kappa}\left[8\left(c_{15} c_{6}+c_{16} c_{7}\right)+\left(c_{6}+c_{7}\right) c_{15}\right]\right) \\
+\varepsilon r_{0}^{2} \cos ^{2} \vartheta \sin ^{2} \varphi\left(\frac{268 C_{1}^{3} \pi^{8}}{\bar{f}_{1}^{3}}+2 \sqrt{\kappa}\left[4\left(c_{15} c_{6}+c_{16} c_{7}\right)+2\left(c_{6}+c_{7}\right) c_{15}\right]\right)
\end{gathered}
$$




$$
\begin{gathered}
+\varepsilon r_{0}^{3} 2 \kappa c_{17}\left(9 \sin ^{2} \vartheta+6 \cos ^{2} \vartheta \sin ^{2} \varphi\right) \\
+\kappa \bar{r}_{0}^{3}\left(c_{11} \sin ^{2} \vartheta \sin ^{2} \varphi+c_{12} \sin ^{2} \vartheta \cos ^{2} \varphi\right) \\
+\kappa \bar{r}_{0}^{3}\left(c_{13} \cos ^{2} \vartheta \sin ^{2} \varphi+c_{14}|\cos \vartheta \sin \vartheta \cos \varphi \sin \varphi|\right) .
\end{gathered}
$$

Now, let us discuss separately the positivity of each summand $I, I I, I I I$. Noting that

$$
I \geq \frac{2 \kappa}{3}|\cos \vartheta \sin \vartheta \cos \varphi \sin \varphi|\left(\sqrt{\left(1+\frac{\bar{r}_{0}^{2}}{10}\right)\left(1+\frac{23 \bar{r}_{0}^{2}}{30}\right)}-\left(1+\frac{2 \bar{r}_{0}^{2}}{5}\right)\right),
$$

we find $I \geq 0$ provided $\bar{r}_{0} \leq \frac{2}{\sqrt{5}}$ which holds if $\varepsilon$ and $\delta$ are (60)-small. Next, we have:

$$
\begin{gathered}
I I \geq \frac{\kappa}{3} \sin ^{2}(\vartheta-\varphi)+\frac{\kappa \bar{r}_{0}^{2}}{180}\left(\sin ^{2} \vartheta+\sin ^{2} \varphi+2 \sin ^{2} \vartheta \sin ^{2} \varphi\right) \\
\quad-\frac{\varepsilon r_{0}}{6}(4|\sin \vartheta|+|\sin (\vartheta-\varphi)|)|\sin (\vartheta-\varphi)|,
\end{gathered}
$$

hence

$$
\begin{gathered}
I I \geq \frac{\kappa}{9} \sin ^{2}(\vartheta-\varphi)+\frac{\kappa \bar{r}_{0}^{2}}{360}\left(\sin ^{2} \vartheta+\sin ^{2} \varphi\right)+\sin ^{2}(\vartheta-\varphi)\left(\frac{\kappa}{9}-\frac{\varepsilon r_{0}}{6}\right) \\
+\frac{\kappa}{9} \sin ^{2}(\vartheta-\varphi)+\frac{\kappa \bar{r}_{0}^{2}}{360} \sin ^{2} \vartheta-\frac{2 \varepsilon r_{0}}{3}|\sin \vartheta \sin (\vartheta-\varphi)| .
\end{gathered}
$$

So, assuming provisionally $I I I \geq 0$, and under the further smallness conditions ${ }^{16}$ :

$$
\varepsilon \delta \leq \frac{2}{3}, \varepsilon \leq \frac{1}{6 \sqrt{10}}
$$

the first of which implies $\left(\frac{\kappa}{9}-\frac{\varepsilon r_{0}}{6}\right) \geq 0$, the second of which ensures that the second line of our last lower bound on $I I$ is identically non-negative, we obtain:

$$
\frac{f_{1}^{3}}{\bar{f}_{1}^{3}} \mathcal{C}\left(m_{0}, V_{0}\right)(\xi, \nu) \geq \frac{\kappa}{9} \sin ^{2}(\vartheta-\varphi)+\frac{\kappa^{2}}{360} r_{0}^{2}\left(\sin ^{2} \vartheta+\sin ^{2} \varphi\right) .
$$

From $r_{0} \leq \bar{r}_{0} \leq \frac{\pi}{2}$ combined with Remark 3 , we find $\frac{f_{1}}{\bar{f}_{1}} \leq \sqrt{\kappa}$, with $\sqrt{\kappa} \leq 1+\frac{\varepsilon}{2} \leq$ $1+\frac{1}{12 \sqrt{10}}$ due to our last smallness assumption on $\varepsilon$. It yields $\frac{f_{1}^{3}}{\bar{f}_{1}^{3}} \leq 1.1$ and the latter, plugged into (27) proves Proposition 2 with $\varsigma=\frac{1}{396}$ in (2).

Finally, let us discuss the non-negativity of III. From $\bar{r}_{0} \leq \frac{\pi}{2}$, we have $\bar{f}_{1}\left(\bar{r}_{0}\right) \geq$ $\frac{2}{\pi}$; moreover, we just saw that $\sqrt{\kappa}$ is bounded above by $1+\frac{1}{12 \sqrt{10}}<1.027$. So

\footnotetext{
${ }^{16}$ implied, for instance, by (51) and (60)
} 
the constants $C_{2}, C_{3}$ defined in Appendix $\mathrm{B}$ can be used as upper bounds on the coefficients respectively of $\varepsilon r_{0}^{2} \sin ^{2} \vartheta$ and $\varepsilon r_{0}^{2} \cos ^{2} \vartheta \sin ^{2} \varphi$ in the lengthy expression which controls $\left|I I I-\frac{\kappa \bar{r}_{0}^{2}}{180} \ldots\right|$ (cf. supra). Using them and recalling (11), we infer from the control just mentionned that:

$$
\begin{aligned}
\frac{1}{r_{0}^{2}} I I I & \geq \sin ^{2} \vartheta\left[\frac{1}{180}-\varepsilon\left(C_{2}+19 c_{17} \delta\right)-\frac{115}{100} \delta\left(c_{11}+c_{12}+\frac{1}{2} c_{14}\right)\right] \\
& +\cos ^{2} \vartheta \sin ^{2} \varphi\left[\frac{1}{180}-\varepsilon\left(C_{3}+13 c_{17} \delta\right)-\frac{115}{100} \delta\left(c_{13}+\frac{1}{2} c_{14}\right)\right] .
\end{aligned}
$$

Therefore $I I I \geq 0$ provided $\varepsilon$ and $\delta$ are taken (61)(62)-small. Proposition 2 is proved.

Concluding the proof of Proposition 2. By inspection of the smallness conditions (59) through (62) which $\varepsilon$ and $\delta_{2}$ must satisfy, we find that (61) is the strongest one bearing on $\varepsilon$, because $C_{2}$ (like $C_{3}<C_{2}$ ) is $O\left(10^{18}\right.$ ) while the constants $c_{i}$ 's (with $11 \leq i \leq 17)$ are $O(1)$. It is also the strongest smallness condition on $\delta=\delta_{2}$ since setting $\varepsilon=1$ in (61) yields $\delta \leq \frac{1}{78}$. We will thus take:

$$
\delta_{2}=0.01
$$

and, plugging this choice in (61), get: $\varepsilon C_{2} \leq 1.214 \times 10^{-3}$. Since $C_{2} \leq 1.4 \times 10^{18}$, it leads us to take:

$$
\eta_{2}=8.6 \times 10^{-22}
$$

So, Proposition 2 holds with $\left(\eta_{2}, \delta_{2}\right)$ as just chosen and $\varsigma_{2}=\frac{1}{396}$ (as found above).

6. $c$-curvature almost-positivity elsewhere. In this section, we prove Theorem 2 at $\left(m_{0}, V_{0}, \xi, \nu\right)$ when $\exp _{m_{0}}\left(V_{0}\right)$ stays away from $m_{0}$ and $m_{0}^{*}$ as specified ${ }^{17}$ in the:

Proposition 3. There exists a couple of small (strictly) positive real numbers $\left(\eta_{3}, \varsigma_{3}\right)$ such that $\mathcal{C}\left(m_{0}, V_{0}\right)(\xi, \nu)$ satisfies the lower bound (2) with $\varsigma=\varsigma_{3}$, provided $\varepsilon=|K-1|_{C^{2}(S)} \leq \eta_{3}$ and $\frac{1}{2} \delta_{2} \leq\left|V_{0}\right| \leq\left(1-\frac{1}{2} \delta_{1}\right) \ell_{0}$.

Proof. The following pinching holds:

$$
\frac{1}{2} \delta_{2} \sqrt{1-\varepsilon} \leq \bar{r}_{0} \leq \pi\left(1-\frac{1}{2} \delta_{1}\right) \sqrt{1+\varepsilon} .
$$

Recalling (26) and assuming that $\varepsilon \leq \eta_{2}$, it implies the other one:

$$
\frac{49}{100} \delta_{2} \leq \bar{r}_{0} \leq\left(1-\frac{1}{4} \delta_{1}\right) \pi,
$$

the right-hand side of which yields the estimate:

$$
\frac{1}{\bar{f}_{1}} \leq \frac{\pi}{\sin \left(\frac{\pi}{4} \delta_{1}\right)},
$$

\footnotetext{
${ }^{17}$ sticking to the notations of Propositions 1 and 2
} 
recorded here for later use. From Corollary 1 combined with (10), (11), $r_{0} \leq \pi$ and $\kappa \geq 1$, we may write:

$$
\begin{aligned}
\frac{f_{1}^{3}}{\bar{f}_{1}^{3}} \mathcal{C}\left(m_{0}, V_{0}\right)(\xi, \nu) & \geq \frac{1}{\kappa} \overline{\mathcal{C}}\left(m_{0}, V_{0}\right)(\xi, \nu)-\frac{\varepsilon}{\bar{f}_{1}^{3}} \sin ^{2} \vartheta\left(338 C_{1}^{3} \pi^{10}+20 \pi\left(c_{6}+c_{7}\right)\right) \\
& -\frac{\varepsilon}{\bar{f}_{1}^{3}} \cos ^{2} \vartheta \sin ^{2} \varphi\left(268 C_{1}^{3} \pi^{10}+20 \pi\left(c_{6}+c_{7}\right)\right)
\end{aligned}
$$

The inequality:

$$
\frac{f_{1}}{\bar{f}_{1}} \leq \frac{\sqrt{\kappa} \sin r_{0}}{\sin \bar{r}_{0}}
$$

obvious from Remark 3, will be used below to deal with the left-hand side of (32). As for the term $\frac{1}{\kappa} \overline{\mathcal{C}}\left(m_{0}, V_{0}\right)(\xi, \nu)$ occurring in the right-hand side of (32), recalling its expression (7), we split it into two summands, namely, the square:

$$
\bar{S}_{1}=2\left(\sin \vartheta \cos \varphi \sqrt{\frac{\bar{r}_{0}^{2}-\sin ^{2} \bar{r}_{0}}{\bar{r}_{0} \sin ^{3} \bar{r}_{0}}}-\cos \theta \sin \varphi \sqrt{\frac{\bar{r}_{0}^{2}-\sin ^{2} \bar{r}_{0}}{\bar{r}_{0}^{3} \sin \bar{r}_{0}}}\right)^{2},
$$

and the remaining part, equal to:

$$
\bar{S}_{2}=\sin ^{2} \vartheta \sin ^{2} \varphi \frac{h_{1}\left(\bar{r}_{0}\right)}{\bar{r}_{0}^{2} \sin ^{2} \bar{r}_{0}}+8 \sin ^{2} \vartheta \cos ^{2} \varphi \frac{\cos \frac{\bar{r}_{0}}{2} h_{2}\left(\frac{\bar{r}_{0}}{2}\right)}{\bar{r}_{0} \sin ^{3} \bar{r}_{0}}+8 \cos ^{2} \vartheta \sin ^{2} \varphi \frac{\cos \frac{\bar{r}_{0}}{2} h_{2}\left(\frac{\bar{r}_{0}}{2}\right)}{\bar{r}_{0}^{3} \sin \bar{r}_{0}}
$$

where

$$
\begin{gathered}
h_{1}(\tau)=\tau^{2}+\tau \sin \tau \cos \tau-2 \sin ^{2} \tau, \\
h_{2}(\tau)=(\tau+\sin \tau \cos \tau) \sin \tau-2 \tau^{2} \cos \tau .
\end{gathered}
$$

Obviously, setting:

$$
\mu_{1}(\tau):=\min \left(\frac{h_{1}(\tau)}{\tau^{2} \sin ^{2} \tau}, \frac{8 \cos \frac{\tau}{2} h_{2}\left(\frac{\tau}{2}\right)}{\tau \sin ^{3} \tau}, \frac{8 \cos \frac{\tau}{2} h_{2}\left(\frac{\tau}{2}\right)}{\tau^{3} \sin \tau}\right),
$$

we have: $\bar{S}_{2} \geq \mu_{1}\left(\bar{r}_{0}\right)\left(\sin ^{2} \vartheta+\cos ^{2} \vartheta \sin ^{2} \varphi\right)$, so we focus on a positive lower bound on $\mu_{1}\left(\bar{r}_{0}\right)$.

To proceed further, let us distinguish two cases and split the proof accordingly.

6.1. First case: $\frac{49}{100} \delta_{2} \leq \bar{r}_{0} \leq 1$. In that case, on the one hand we may write:

$$
\frac{1}{\bar{f}_{1}} \leq \frac{1}{\sin 1}
$$

on the other hand, combining (33) with the alternating series test applied to the Maclaurin series of $\sin \bar{r}_{0}$, we get:

$$
\frac{f_{1}}{\bar{f}_{1}} \leq \frac{1}{1-\frac{\bar{r}_{0}^{2}}{6}} \leq \frac{6}{5}
$$


so (32) implies:

$$
\left(\frac{6}{5}\right)^{3} \mathcal{C}\left(m_{0}, V_{0}\right)(\xi, \nu) \geq \frac{1}{\kappa} \overline{\mathcal{C}}\left(m_{0}, V_{0}\right)(\xi, \nu)-\frac{\varepsilon C_{4}}{\sin ^{3} 1}\left(\sin ^{2} \vartheta+\cos ^{2} \vartheta \sin ^{2} \varphi\right)
$$

where $C_{4}$ is the constant defined in Appendix B.4. Besides, the following lemma holds:

LEMma 3. The function $h_{1}$ (resp. $\left.h_{2}\right)$ is increasing on $[0, \pi]$ (resp. on $\left.\left[0, \frac{\pi}{2}\right]\right)$. Furthermore, for each $\tau \in[0,1]$, the alternating series test holds for the Maclaurin series of $h_{1}(\tau)$ and $h_{2}(\tau)$, implying the lower bounds:

$$
h_{1}(\tau) \geq \frac{2}{315} \tau^{6}\left(7-\tau^{2}\right), h_{2}(\tau) \geq \frac{4}{5} \tau^{6}\left(\frac{2}{9}-\frac{1}{21} \tau^{2}\right) .
$$

The proof is lengthy but rather elementary hence left as an exercise. Combining this lemma with the standard bounds $\sin \tau \leq \tau, \cos \frac{\tau}{2} \geq 1-\frac{\tau^{2}}{8}$, we get:

$$
\mu_{1}\left(\bar{r}_{0}\right) \geq \min \left(\frac{14}{315} \bar{r}_{0}^{2}\left(1-\frac{1}{7} \bar{r}_{0}^{2}\right), \frac{1}{45} \bar{r}_{0}^{2}\left(1-\frac{5}{28} \bar{r}_{0}^{2}\right)\right) \equiv \frac{1}{45} \bar{r}_{0}^{2}\left(1-\frac{5}{28} \bar{r}_{0}^{2}\right)
$$

and, from (30), conclude: $\mu_{1}\left(\bar{r}_{0}\right) \geq 4.38 \times 10^{-3} \delta_{2}^{2}$. This lower bound combined with (35) implies:

$$
\mathcal{C}\left(m_{0}, V_{0}\right)(\xi, \nu) \geq\left(\frac{5}{6}\right)^{3} 2.19 \times 10^{-3} \delta_{2}^{2}\left(\sin ^{2} \vartheta+\cos ^{2} \vartheta \sin ^{2} \varphi\right)
$$

provided $\varepsilon$ is taken (63)-small. Recalling (21), we thus obtain (2) at $\left(m_{0}, V_{0}, \xi, \nu\right)$ with $\varsigma=\frac{1}{4 \pi^{2}}\left(\frac{5}{6}\right)^{3} 2.19 \times 10^{-3} \delta_{2}^{2}$. Here, the value of $\delta_{2}$ is the one chosen at the end of Section 5 , namely $\delta_{2}=0.01$; plugging it in the preceding formula, and in (63) together with the sharp bound $C_{4} \leq 3.6 \times 10^{18}$, leads us to take:

$$
\eta_{3} \leq 3.62 \times 10^{-26}, \varsigma_{3}=3.21 \times 10^{-9} .
$$

6.2. Second case: $1 \leq \bar{r}_{0} \leq\left(1-\frac{\delta_{1}}{4}\right) \pi$. Back to (32), using (31), we now have:

$$
\frac{f_{1}^{3}}{\bar{f}_{1}^{3}} \mathcal{C}\left(m_{0}, V_{0}\right)(\xi, \nu) \geq \frac{1}{\kappa} \overline{\mathcal{C}}\left(m_{0}, V_{0}\right)(\xi, \nu)-\frac{\varepsilon \pi^{3} C_{4}}{\sin ^{3}\left(\frac{\delta_{1}}{4} \pi\right)}\left(\sin ^{2} \vartheta+\cos ^{2} \vartheta \sin ^{2} \varphi\right)
$$

hence:

$$
\frac{f_{1}^{3}}{\bar{f}_{1}^{3}} \mathcal{C}\left(m_{0}, V_{0}\right)(\xi, \nu) \geq\left(\mu_{1}\left(\bar{r}_{0}\right)-\frac{\varepsilon \pi^{3} C_{4}}{\sin ^{3}\left(\frac{\delta_{1}}{4} \pi\right)}\right)\left(\sin ^{2} \vartheta+\cos ^{2} \vartheta \sin ^{2} \varphi\right)
$$

Moreover, using:

$$
\sin \tau=\sin (\pi-\tau) \leq \pi-\tau, \text { and } \cos \frac{\tau}{2}=\sin \frac{(\pi-\tau)}{2} \geq \frac{(\pi-\tau)}{2}-\frac{(\pi-\tau)^{3}}{48}
$$


with $\tau=\bar{r}_{0}$, we have:

$$
\frac{\cos \frac{\bar{r}_{0}}{2}}{\sin \bar{r}_{0}} \geq \frac{1}{2}-\frac{(\pi-1)^{2}}{48} \geq 0.4
$$

therefore:

$$
\frac{8 \cos \frac{\bar{r}_{0}}{2} h_{2}\left(\frac{\bar{r}_{0}}{2}\right)}{\bar{r}_{0}^{3} \sin \bar{r}_{0}} \geq \frac{3.2}{\pi^{3}} h_{2}\left(\frac{1}{2}\right) \geq 2.6 \times 10^{-4} .
$$

Besides, we directly get:

$$
\frac{h_{1}\left(\bar{r}_{0}\right)}{\bar{r}_{0}^{2} \sin ^{2} \bar{r}_{0}} \geq \frac{h_{1}(1)}{\pi^{2}} \geq 3.9 \times 10^{-3},
$$

and thus conclude: $\mu_{1}\left(\bar{r}_{0}\right) \geq 2.6 \times 10^{-4}$. Finally, from (33), we infer the bound:

$$
\frac{f_{1}}{\bar{f}_{1}} \leq\left(1+\frac{\varepsilon}{2}\right) \frac{\sin r_{0}}{\sin \bar{r}_{0}},
$$

and from the identity:

$$
\sin r_{0} \equiv \sin \bar{r}_{0} \cos \left[(\sqrt{\kappa}-1) r_{0}\right]-\cos \bar{r}_{0} \sin \left[(\sqrt{\kappa}-1) r_{0}\right],
$$

we readily get:

$$
\frac{\sin r_{0}}{\sin \bar{r}_{0}} \leq 1+\frac{\sin \left[(\sqrt{\kappa}-1) r_{0}\right]}{\sin \left(\frac{\delta_{1}}{4} \pi\right)} \leq 1+\frac{2 \varepsilon}{\delta_{1}\left(1-\frac{\delta_{1}^{2} \pi^{2}}{96}\right)} .
$$

It prompts us to take $\varepsilon(64)$-small with $\delta_{1}$ as chosen at the end of Section 4 (namely $\left.\delta_{1}=1.48 \times 10^{-15}\right)$, in order to keep the ratio $\frac{f_{1}}{\bar{f}_{1}}$ below $\frac{6}{5}$. Plugging in (37) the latter upper bound together with the former lower bound on $\mu_{1}\left(\bar{r}_{0}\right)$, we obtain:

$$
\mathcal{C}\left(m_{0}, V_{0}\right)(\xi, \nu) \geq\left(\frac{5}{6}\right)^{3} 1.3 \times 10^{-4}\left(\sin ^{2} \vartheta+\cos ^{2} \vartheta \sin ^{2} \varphi\right)
$$

provided $\varepsilon$ is taken (65)-small. Recalling (21), we conclude that $\mathcal{C}\left(m_{0}, V_{0}\right)(\xi, \nu)$ satisfies (2) with:

$$
\varsigma \leq \frac{1}{4 \pi^{2}}\left(\frac{5}{6}\right)^{3} 1.3 \times 10^{-4}
$$

so here, it is sufficient to take: $\varsigma_{3} \leq 1.9 \times 10^{-6}$, a condition well satisfied by the value chosen in (36) for $\varsigma_{3}$. Finally, recalling that $\delta_{1}$ was taken equal to $1.48 \times 10^{-15}$, the smallness condition (65) on $\varepsilon$ leads us to take:

$$
\eta_{3}=1.8 \times 10^{-69} .
$$

This tiny value (compare with (26)(29)(36)) reflects the fact that a perturbation device from the constant curvature case becomes outrageously rough as $\left|V_{0}\right| \uparrow \ell_{0}$ (i.e. getting close to the first conjugate point). 
7. Proof of Theorem 2. The proof of Theorem 2 at $\left(m_{0}, V_{0}, \xi, \nu\right)$ goes by combining Propositions 1, 2 and 3. Doing so, we first observe that the assumption made on $\left|V_{0}\right|$ in Proposition 3 overlaps, as it should, the corresponding ones of Propositions 1 and 2 . Next, since $\varepsilon$ should now fulfill all the smallness conditions stated on it in Sections 4, 5 and 6 , we take $\eta$ in the statement of Theorem 2 equal to:

$$
\eta=\min \left(\eta_{1}, \eta_{2}, \eta_{3}\right) \equiv \eta_{3}=1.8 \times 10^{-69} .
$$

Similarly, we choose:

$$
\varsigma=\min \left(\varsigma_{1}, \varsigma_{2}, \varsigma_{3}\right) \equiv \varsigma_{3}=3.21 \times 10^{-9}
$$

Appendix A. Proof of Lemma 1. We will proceed stepwise in the Fermi chart along $V_{0}$, using repeatedly the Maclaurin theorem, the solution map $\mathcal{S}_{\bar{r}_{0}}$ and its contraction property, to derive estimates at $\left(\left(0, r_{0}\right), t\right)$, uniform in $t \in[0,1]$, on the expressions appearing in the conclusion of Lemma 1 and also on $\left|D^{2} X\right|$ and $\left|D^{j} \mathcal{K}\right|$ for $j=1,2$, where $\mathcal{K}=K \circ X$.

\section{A.1. Estimates of order 0 .}

A.1.1. Basic estimates. From Remark 3, we may take $B_{101}=1$. Besides, we have:

$$
\|\kappa-\mathcal{K}\| \leq \varepsilon \min \left(1, r_{0}\right)
$$

On the axis of the Fermi chart, the functions $\tilde{f}_{a}=f_{a}-\bar{f}_{a}$ (with $a=0,1$ ) satisfy:

$$
\frac{d^{2} \tilde{f}_{a}}{d t^{2}}+r_{0}^{2} \kappa \tilde{f}_{a}=\phi_{0 a} \text { with } \phi_{0 a}=r_{0}^{2}(\kappa-\mathcal{K}) f_{a} .
$$

Combining the latter with (8) applied to $\mathcal{S}_{\bar{r}_{0}}$, and (39), we get:

$$
\left\|\tilde{f}_{a}\right\| \leq \frac{\varepsilon}{2} \min \left(r_{0}^{2}, r_{0}^{3}\right)\left\|f_{a}\right\| .
$$

If $a=0$, since $\left\|f_{0}\right\| \leq\left\|\tilde{f}_{0}\right\|+\left\|\bar{f}_{0}\right\| \leq\left\|\tilde{f}_{0}\right\|+1$, we infer:

$$
\left\|\tilde{f}_{0}\right\| \leq \frac{\mu}{1-\mu} \text { with } \mu=\frac{\varepsilon}{2} \min \left(r_{0}^{2}, r_{0}^{3}\right)
$$

while if $a=1$, recalling Remark 3, we get at once: $\left\|\tilde{f}_{1}\right\| \leq \mu$. Since $\varepsilon \leq \frac{1}{\pi^{2}}$, we have $\varepsilon r_{0}^{2} \leq 1$ (recalling Remark 2), an inequality used throughout this appendix. So we readily obtain:

$$
\left\|\tilde{f}_{0}\right\| \leq \varepsilon \min \left(r_{0}^{2}, \frac{r_{0}^{3}}{2-\varepsilon r_{0}^{3}}\right) .
$$

In particular, regarding the first line of conclusion of the lemma for $k=0$, we may take $B_{200}=1, B_{201}=\frac{1}{2}$, which yields $B_{100}=2$ after use of the triangle inequality. Similarly, setting $h_{0}=1$ and $h_{1}=t$, we find on the axis:

$$
f_{a}-h_{a}=\mathcal{S}_{\overline{r_{0}}}\left(-r_{0}^{2} \mathcal{K} h_{a}+r_{0}^{2}(\kappa-\mathcal{K})\left(f_{a}-h_{a}\right)\right)
$$


for $a=0,1$. Combining (8) with an argument as the one above for $\tilde{f}_{0}$ yields:

$$
\left\|f_{a}-h_{a}\right\| \leq \frac{r_{0}^{2}\|\mathcal{K}\|}{2-\varepsilon r_{0}^{2}} \leq r_{0}^{2}\|\mathcal{K}\|
$$

hence the inequalities:

$$
\left\|f_{0}-1\right\| \leq r_{0}^{2}(1+\varepsilon),\left\|f_{1}-t\right\| \leq r_{0}^{2}(1+\varepsilon),
$$

recorded here for later use.

A.1.2. Estimates on Maclaurin approximations. The first order Maclaurin approximation of $\mathcal{K}$ at $t=0$ satisfies the estimate:

$$
\left\|\mathcal{K}-\kappa-t r_{0} \partial_{2} K(0)\right\| \leq \frac{1}{2} \varepsilon r_{0}^{2} .
$$

The latter combined with the triangle inequality is used to evaluate the remainder of the first non trivial Maclaurin approximation of $\phi_{0 a}$ at $t=0$, namely of $\phi_{0 a}+$ $t^{a+1} r_{0}^{3} \partial_{2} K(0)$ written as:

$$
\phi_{0 a}+t^{a+1} r_{0}^{3} \partial_{2} K(0)=-r_{0}^{2}\left(\mathcal{K}-\kappa-t r_{0} \partial_{2} K(0)\right) f_{a}+t r_{0}^{3} \partial_{2} K(0)\left(h_{a}-f_{a}\right) .
$$

It leads us to the upper bound:

$$
\left\|\phi_{0 a}+t^{a+1} r_{0}^{3} \partial_{2} K(0)\right\| \leq \frac{1}{2} \varepsilon r_{0}^{4}\left\|f_{a}\right\|+\varepsilon r_{0}^{3}\left\|f_{a}-h_{a}\right\|
$$

which, combined with (40) and (8), yields for

$$
\tilde{f}_{a}+r_{0}^{3} \psi_{0} \mathcal{S}_{\overline{r_{0}}}\left(t^{a+1}\right) \equiv \mathcal{S}_{\overline{r_{0}}}\left(\phi_{0 a}+t^{a+1} r_{0}^{3} \partial_{2} K(0)\right)
$$

the desired second line of conclusion with $B_{30 a}=\frac{1}{4} B_{10 a}+\frac{\pi}{2}\left(1+\frac{1}{\pi^{2}}\right)$.

\section{A.2. Estimates of order 1 .}

A.2.1. Basic estimates. From the definition of $\mathcal{K}$ and $f_{1}$, we have at $\left(v_{0}, t\right)$ :

$$
D_{1} \mathcal{K}=f_{1}(t)\left(\partial_{1} K\right)\left(0, t r_{0}\right), D_{2} \mathcal{K}=t\left(\partial_{2} K\right)\left(0, t r_{0}\right)
$$

Recalling Remark 3, we conclude:

$$
\forall i=1,2, \forall t \in[0,1],\left|D_{i} \mathcal{K}\left(v_{0}, t\right)\right| \leq \varepsilon, \text { thus }\left\|D_{\nu} \mathcal{K}\left(v_{0}, .\right)\right\| \leq \sqrt{2} \varepsilon .
$$

Besides, if we apply $D_{\nu}$ to the Jacobi equations:

$$
\ddot{f}+|v|^{2} \mathcal{K}(v, t) f=0 \text { and } \ddot{f}+|v|^{2} \kappa f=0,
$$

then let $v=v_{0}=\left(0, r_{0}\right)$, we readily infer for $\tilde{f}_{a}$ the equation (still abbreviating freely $D_{\nu}$ by a prime): $\frac{d^{2} \tilde{f}_{a}^{\prime}}{d t^{2}}+r_{0}^{2} \kappa \tilde{f}_{a}^{\prime}=\phi_{1 a}$, with:

$$
\phi_{1 a}=r_{0}^{2}(\kappa-\mathcal{K}) f_{a}^{\prime}-2 r_{0} \cos \varphi \kappa \tilde{f}_{a}+2 r_{0} \cos \varphi f_{a}(\kappa-\mathcal{K})-r_{0}^{2} \mathcal{K}^{\prime} f_{a}
$$


and for $\bar{f}_{a}$ the equation: $\frac{d^{2} \bar{f}_{a}^{\prime}}{d t^{2}}+r_{0}^{2} \kappa \bar{f}_{a}^{\prime}=-2 r_{0} \cos \varphi \kappa \bar{f}_{a}$. Recalling (8), we get from the latter the auxiliary bound:

$$
\left\|\bar{f}_{a}^{\prime}\right\| \leq r_{0} \kappa \leq c_{1}
$$

(see Appendix B), and from the former:

$$
\left\|\tilde{f}_{a}^{\prime}\right\| \leq \frac{1}{2} r_{0}^{2}\|\kappa-\mathcal{K}\|\left(\left\|\tilde{f}_{a}^{\prime}\right\|+\left\|\bar{f}_{a}^{\prime}\right\|\right)+r_{0} \kappa\left\|\tilde{f}_{a}\right\|+r_{0}\|\kappa-\mathcal{K}\|\left\|f_{a}\right\|+\frac{1}{2} r_{0}^{2}\left\|\mathcal{K}^{\prime}\right\|\left\|f_{a}\right\|,
$$

after use of the triangle inequality. Previous bounds, namely (39)(42)(44) and those of Lemma 1 for $k=0$, yield:

$$
\left\|\tilde{f}_{a}^{\prime}\right\| \leq \frac{1}{1-\frac{1}{2} \varepsilon r_{0}^{2}}\left(\frac{1}{2} \varepsilon \kappa r_{0}^{3}+B_{20 a} \varepsilon \kappa r_{0}^{3}+B_{10 a} \varepsilon r_{0}+\frac{1}{2} B_{10 a} \sqrt{2} \varepsilon r_{0}^{2}\right)
$$

hence the conclusion of the first line of the lemma holds for $k=1$ with:

$$
B_{21 a}=1+\pi^{2}+2 B_{20 a}\left(1+\pi^{2}\right)+B_{10 a}(2+\pi \sqrt{2}),
$$

and, combining the triangle inequality with the auxiliary bound on $\bar{f}_{a}^{\prime}$, with:

$$
B_{11 a}=\pi+\frac{1}{\pi}\left(1+B_{21 a}\right) \text {. }
$$

A.2.2. Estimates on Maclaurin approximations. From the expression found above for $D \mathcal{K}\left(v_{0}, t\right)$, we may write:

$$
D_{\nu} \mathcal{K}\left(v_{0}, t\right)=t \partial_{\nu} K\left(0, t r_{0}\right)+\sin \varphi \partial_{1} K\left(0, t r_{0}\right)\left(f_{1}-t\right) .
$$

So, using the straightforward bound: $\left|\partial_{\nu} K\left(0, t r_{0}\right)-\partial_{\nu} K(0)\right| \leq \varepsilon r_{0}$ combined with the triangle inequality and (40), we obtain:

$$
\left\|\mathcal{K}^{\prime}-t \partial_{\nu} K(0)\right\| \leq\left(1+c_{1}\right) \varepsilon r_{0} .
$$

We wish now to estimate the remainder of the first non trivial Maclaurin approximation of $\phi_{1 a}$ at $t=0$, namely the $\|$.$\| norm of the expression:$

$$
\phi_{1 a}+2 t h_{a} r_{0}^{2} \cos \varphi \partial_{2} K(0)+t h_{a} r_{0}^{2} \partial_{\nu} K(0) .
$$

To do so, we recast the latter as follows:

$$
\begin{gathered}
=-2 \kappa r_{0} \cos \varphi \tilde{f}_{a}-2 r_{0} \cos \varphi f_{a}\left(\mathcal{K}-\kappa-t r_{0} \partial_{2} K(0)\right)+2 t r_{0}^{2} \cos \varphi \partial_{2} K(0)\left(h_{a}-f_{a}\right) \\
+r_{0}^{2}(\kappa-\mathcal{K})\left(\tilde{f}_{a}^{\prime}+\bar{f}_{a}^{\prime}\right)-r_{0}^{2}\left(\mathcal{K}^{\prime}-t \partial_{\nu} K(0)\right) f_{a}-t r_{0}^{2} \partial_{\nu} K(0)\left(f_{a}-h_{a}\right)
\end{gathered}
$$

and apply the triangle inequality combined with (39)(40)(41)(44)(45) and the bounds of the lemma on the $\|$.$\| norms of \tilde{f}_{a}, \tilde{f}_{a}^{\prime}$. Observing that, if we apply the map $\mathcal{S}_{\overline{r_{0}}}$ to the preceding expression and use (9), we recover $\tilde{f}_{a}^{\prime}+r_{0}^{2} \psi_{1} \mathcal{S}_{\overline{r_{0}}}\left(t^{a+1}\right)$, and recalling (8), we infer that $\left\|\tilde{f}_{a}^{\prime}+r_{0}^{2} \psi_{1} \mathcal{S}_{\overline{r_{0}}}\left(t^{a+1}\right)\right\|$ is bounded above by:

$$
\varepsilon r_{0}^{3}\left(\frac{1}{2} B_{10 a}\left(2+\pi+\frac{1}{\pi}\right)+B_{20 a} \kappa+B_{21 a} \frac{\varepsilon}{2}+\frac{3}{2} r_{0}(1+\varepsilon)+\frac{\kappa}{2}\right) .
$$

The second line of conclusion of Lemma 1 for $k=1$, indeed, follows with:

$$
B_{31 a}=\frac{1}{2} B_{10 a}\left(2+\pi+\frac{1}{\pi}\right)+B_{20 a}\left(1+\frac{1}{\pi^{2}}\right)+B_{21 a} \frac{1}{2 \pi^{2}}+\frac{(3 \pi+1)}{2}\left(1+\frac{1}{\pi^{2}}\right) .
$$




\section{A.3. Estimates of order 2.}

A.3.1. Basic estimates. As in [7], applying twice $D_{\nu}$ to the geodesic equation with initial conditions $(0, v)$, then letting $v=v_{0}=\left(0, r_{0}\right)$, and recalling the 2-dimensional formulas given after Definition 2 for the derivatives of the Christoffel symbols on the axis of the Fermi chart, yields for $D_{\nu \nu} X^{i}(t)=D_{\nu \nu} X^{i}\left(0, v_{0}, t\right)$ the following equations, with zero initial conditions:

$$
\begin{aligned}
\frac{d^{2}}{d t^{2}}\left(D_{\nu \nu} X^{1}\right)+r_{0}^{2} \mathcal{K} D_{\nu \nu} X^{1}= & -4 r_{0} \cos \varphi \sin \varphi \mathcal{K} f_{1} \\
& -r_{0}^{2} \sin ^{2} \varphi f_{1}^{2}\left(\left(\partial_{1} K\right) \circ X\right) \\
& -2 r_{0}^{2} \sin \varphi \cos \varphi t f_{1}\left(\left(\partial_{2} K\right) \circ X\right), \\
\frac{d^{2}}{d t^{2}}\left(D_{\nu \nu} X^{2}\right)=4 r_{0} \sin ^{2} \varphi \mathcal{K} & f_{1} \dot{f}_{1}+r_{0}^{2} \sin ^{2} \varphi f_{1}^{2}\left(\left(\partial_{2} K\right) \circ X\right) .
\end{aligned}
$$

To treat the first equation, we view $\mathcal{K}$ as a perturbation of $\kappa$ and apply the solution map $\mathcal{S}_{\bar{r}_{0}}$ and the estimates (8) (39) and that on $\left\|f_{1}\right\|$; to treat the second equation, we use our estimates on $\|\mathcal{K}\|$ and $\left\|f_{1}\right\|$ and note the further one:

$$
\dot{f}_{1}(t)=1+\int_{0}^{t} \ddot{f}_{1}(\theta) d \theta \equiv 1-r_{0}^{2} \int_{0}^{t} \mathcal{K}(\theta) f_{1}(\theta) d \theta \Longrightarrow\left\|\dot{f}_{1}\right\| \leq 1+r_{0}^{2}(1+\varepsilon) \leq 2+\pi^{2} .
$$

We readily find:

$$
\left\|D_{\nu \nu} X^{1}\right\| \leq c_{2} r_{0},\left\|D_{\nu \nu} X^{2}\right\| \leq c_{3} r_{0} .
$$

Next, we calculate the expression of $D_{\nu \nu} \mathcal{K}\left(v_{0}, t\right)$ and obtain:

$$
\begin{aligned}
D_{\nu \nu} \mathcal{K}\left(v_{0}, t\right)= & \partial_{1} K\left(0, t r_{0}\right) D_{\nu \nu} X^{1}+\partial_{2} K\left(0, t r_{0}\right) D_{\nu \nu} X^{2} \\
& +\partial_{11} K\left(0, t r_{0}\right) f_{1}^{2} \sin ^{2} \varphi+2 \partial_{12} K\left(0, t r_{0}\right) t f_{1} \sin \varphi \cos \varphi \\
& +\partial_{22} K\left(0, t r_{0}\right) t^{2} \cos ^{2} \varphi
\end{aligned}
$$

from what we infer, using (46) combined with Remark 3 :

$$
\left\|D_{\nu \nu} \mathcal{K}\left(v_{0}, .\right)\right\| \leq\left(c_{2}+c_{3}\right) \varepsilon r_{0}+2 \varepsilon \leq c_{4} \varepsilon .
$$

Now, we apply $D_{\nu \nu}$ to (43) and get, on the one hand:

$$
\frac{d^{2}}{d t^{2}}\left(\bar{f}_{a}^{\prime \prime}\right)+r_{0}^{2} \kappa \bar{f}_{a}^{\prime \prime}=-2 \kappa \bar{f}_{a}-4 \kappa r_{0} \cos \varphi \bar{f}_{a}^{\prime},
$$

from what, recalling (44), we infer the auxiliary bound:

$$
\left\|\bar{f}_{a}^{\prime \prime}\right\| \leq c_{5}
$$

on the other hand:

$$
\frac{d^{2}}{d t^{2}}\left(\tilde{f}_{a}^{\prime \prime}\right)+r_{0}^{2} \kappa \tilde{f}_{a}^{\prime \prime}=\phi_{2 a}
$$


with:

$$
\begin{aligned}
\phi_{2 a}= & r_{0}^{2}(\kappa-\mathcal{K}) f_{a}^{\prime \prime}+2\left(\kappa \bar{f}_{a}-\mathcal{K} f_{a}\right)+4 r_{0} \cos \varphi\left(\kappa \bar{f}_{a}^{\prime}-\mathcal{K} f_{a}^{\prime}\right) \\
& -4 r_{0} \cos \varphi f_{a} D_{\nu} \mathcal{K}-2 r_{0}^{2} f_{a}^{\prime} D_{\nu} \mathcal{K}-r_{0}^{2} f_{a} D_{\nu \nu} \mathcal{K}
\end{aligned}
$$

Finally, from (8) applied (with $\omega=\bar{r}_{0}$ ) to the latter equation, we routinely derive the first line of conclusion of Lemma 1 for $k=2$ with:

$$
B_{12 a}=c_{5}+\frac{1}{\pi^{2}} B_{22 a},
$$

after use of the triangle inequality combined with (48), and:

$B_{22 a}=6+\pi^{2}\left(4+c_{5}\right)+\left(4 \sqrt{2}+\pi c_{4}\right) \pi B_{10 a}+2 \sqrt{2} \pi^{2} B_{11 a}+2\left(1+\pi^{2}\right)\left(B_{20 a}+2 B_{21 a}\right)$,

after use of the triangle inequality combined with (39)(42)(44)(47) and the bounds of the same line of conclusion for $k=0,1$.

A.3.2. Estimates on Maclaurin approximations. Finally, in order to estimate the $\|$.$\| norm of \tilde{f}_{a}^{\prime \prime}+r_{0} \psi_{2} \mathcal{S}_{\bar{r}_{0}}\left(t^{a+1}\right)$, we note that the latter is equal to $\mathcal{S}_{\bar{r}_{0}}\left(\phi_{2 a}+2 t h_{a} r_{0} \partial_{2} K(0)+4 r_{0} \cos \varphi t h_{a} \partial_{\nu} K(0)\right)$, we recast the argument of $\mathcal{S}_{\bar{r}_{0}}$ as follows:

$$
\begin{aligned}
\phi_{2 a}+ & 2 t h_{a} r_{0} \partial_{2} K(0)+4 r_{0} \cos \varphi t h_{a} \partial_{\nu} K(0)=r_{0}^{2}(\kappa-\mathcal{K}) f_{a}^{\prime \prime}-2 r_{0}^{2} f_{a}^{\prime} D_{\nu} \mathcal{K}-r_{0}^{2} f_{a} D_{\nu \nu} \mathcal{K} \\
& -2 \kappa \tilde{f}_{a}-2 t r_{0} \partial_{2} K(0)\left(f_{a}-h_{a}\right)-2\left(\mathcal{K}-\kappa-t r_{0} \partial_{2} K(0)\right) f_{a}-4 r_{0} \cos \varphi \kappa \tilde{f}_{a}^{\prime}
\end{aligned}
$$$$
+4 r_{0} \cos \varphi(\kappa-\mathcal{K}) f_{a}^{\prime}-4 r_{0} \cos \varphi f_{a}\left(D_{\nu} \mathcal{K}-t \partial_{\nu} K(0)\right)+4 t r_{0} \cos \varphi\left(h_{a}-f_{a}\right) \partial_{\nu} K(0),
$$

and we apply (8) with $\omega=\bar{r}_{0}$ to the right-hand expression, combined with the triangle inequality, the previous bounds of Lemma 1 and (39) (40)(41)(42)(45)(47). Doing so term by term, we obtain the second line of conclusion of Lemma 1 for $k=2$ with:

$$
\begin{aligned}
B_{32 a}= & \frac{1}{2} B_{12 a}+\sqrt{2} B_{11 a}+\frac{1}{2} B_{10 a} c_{4}+\left(1+\frac{1}{\pi^{2}}\right) B_{20 a}+c_{1} \\
& +\frac{1}{2} B_{10 a}+2\left(1+\frac{1}{\pi^{2}}\right) B_{21 a}+2 B_{11 a}+2 B_{10 a}\left(1+c_{1}\right)+2 c_{1} \\
\equiv & 3 c_{1}+\frac{1}{2}\left(5+4 c_{1}+c_{4}\right) B_{10 a}+(\sqrt{2}+2) B_{11 a}+\frac{1}{2} B_{12 a} \\
& +\left(1+\frac{1}{\pi^{2}}\right)\left(B_{20 a}+2 B_{21 a}\right) .
\end{aligned}
$$

\section{Appendix B. Auxiliary universal constants and conditions.}

\section{B.1. List of constants for Section 3.}

$$
\begin{gathered}
c_{1}=\pi+\frac{1}{\pi}, c_{2}=4\left(\frac{3}{4 \pi}+\frac{1}{\pi^{2}}+1\right), c_{3}=\frac{1}{2 \pi}+2\left(1+\frac{1}{\pi^{2}}\right)\left(2+\pi^{2}\right), \\
c_{4}=\frac{11}{2}+10 \pi+\frac{8}{\pi}+2 \pi^{3}, c_{5}=1+\frac{1}{\pi^{2}}+2 c_{1}^{2},
\end{gathered}
$$




$$
\begin{gathered}
c_{6}=\sup _{\tau \in[0,2 \pi]}\left|\frac{\tau-\sin \tau}{\tau^{3}}\right|, c_{7}=\sup _{\tau \in[0,2 \pi]}\left|\frac{\tau^{2}+2(\cos \tau-1)}{\tau^{4}}\right|, \\
c_{8}=\sup _{\tau \in[0,2 \pi]}\left|\frac{\tau \cos \tau-\sin \tau}{\tau^{2}}\right|, c_{9}=\sup _{\tau \in[0,2 \pi]}\left|\frac{\tau \cos \tau-\sin \tau}{\tau^{3}}\right|, \\
c_{10}=\sup _{\tau \in[0,2 \pi]}\left|\frac{\cos \tau \sin \tau-\tau}{\tau^{3}}\right|, \\
C_{1}=\max \left(\max _{a=0,1 ; k=0,1,2}\left(B_{1 k a}, B_{3 k a}\right), 8 c_{6}, 8 c_{7}, \frac{19}{18} c_{8}, \frac{10}{9} c_{9}, c_{10}\right) .
\end{gathered}
$$

B.2. List of conditions on $\varepsilon$ and $\delta=\delta_{1}$ for Section 4 .

$$
\frac{|\cos \varphi|}{\pi}\left(1-\frac{\frac{\varepsilon}{2}}{1-\left(\frac{\varepsilon}{2}+\delta\right)}-\frac{\pi^{2}}{2}\left(\frac{\varepsilon}{2}+\delta\right)^{2}\right)-\varepsilon \pi B_{211}>0
$$

an inequality to be used only in subsection 4.2 with $|\cos \varphi|$ replaced by $\frac{1}{7704}$;

$$
\begin{aligned}
& \frac{2 \varepsilon}{1-\left(\frac{\varepsilon}{2}+\delta\right)}+2 \pi^{2}\left(\frac{\varepsilon}{2}+\delta\right)^{2}+\frac{\varepsilon \pi^{2}}{2}\left(1+\frac{\varepsilon}{2}\right)+\frac{3\left(\frac{\varepsilon}{2}+\delta\right)}{1-\left(\frac{\varepsilon}{2}+\delta\right)} \leq \frac{1}{2} \\
\frac{1}{24 \pi^{2}} \geq & R_{1}(\varepsilon, \delta):=\varepsilon\left(B_{221}+\frac{1}{8} B_{200}\right)+\frac{1}{16}\left(\frac{\varepsilon}{2}+\delta\right)^{2} \\
+ & 2 \pi B_{111}\left(\left(\frac{\varepsilon}{2}+\delta\right)\left(1+\frac{\varepsilon}{2}\right)+\varepsilon B_{210}\right) \\
+ & \frac{\frac{\varepsilon}{2}+\delta}{1-\left(\frac{\varepsilon}{2}+\delta\right)}\left[B_{120}+\frac{2}{\pi^{2}\left(1-\frac{\varepsilon}{2}-\delta\right)^{2}}\left(1+\varepsilon \pi^{2} B_{200}+\frac{\frac{\varepsilon}{2}+\delta}{1-\left(\frac{\varepsilon}{2}+\delta\right)}\right)\right]
\end{aligned}
$$

$$
\frac{1}{16 \sqrt{3}\left(\pi^{2}+5\right)} \geq \sqrt{\frac{\frac{\varepsilon}{2}+\delta}{1-\left(\frac{\varepsilon}{2}+\delta\right)}}
$$

$$
\begin{aligned}
\frac{1}{24 \pi^{2}} \geq R_{2}(\varepsilon, \delta):= & R_{1}(\varepsilon, \delta)+8 B_{111}\left(\left(\frac{\varepsilon}{2}+\delta\right)\left(1+\frac{\varepsilon}{2}\right) \pi+\varepsilon \pi B_{210}\right) \\
& \varepsilon \leq \frac{1}{\pi^{2} B_{211}}\left(\frac{1}{\pi \sqrt{192}}-\frac{1}{3852}\right)
\end{aligned}
$$

$$
\begin{gathered}
\frac{1}{15408 \pi} \geq \varepsilon \pi B_{211}+\frac{\frac{\varepsilon}{2}}{\pi\left(1-\left(\frac{\varepsilon}{2}+\delta\right)\right)}+\left(\frac{\varepsilon}{2}+\delta\right)^{2} \frac{\pi}{2} \\
\frac{1}{2 \pi^{2} 15408^{2}} \geq \frac{\frac{\varepsilon}{2}+\delta}{1-\left(\frac{\varepsilon}{2}+\delta\right)}\left(B_{120}+\frac{3}{2} B_{121}+2 B_{110} B_{111}+\frac{36}{5 \pi^{2}}\right)
\end{gathered}
$$




$$
\frac{1}{100 \pi^{2} 15408^{2}} \geq \frac{\frac{\varepsilon}{2}+\delta}{1-\left(\frac{\varepsilon}{2}+\delta\right)}\left(B_{120}+\frac{3}{2} B_{121}+2 B_{110} B_{111}+\frac{36}{5 \pi^{2}}\right)
$$

(58) $\frac{\sqrt{3}}{8 \pi^{2}} \geq \frac{\left(\frac{\varepsilon}{2}+\delta\right)}{\left(1-\frac{\varepsilon}{2}-\delta\right)^{3} \pi^{2}}\left(\frac{6}{5}+\left(1+\frac{\varepsilon}{2}\right)^{2} \pi^{2}\right)+\varepsilon\left(B_{221}+\frac{16}{5} B_{211}\right)$

$$
+7 \pi B_{111}\left(\left(\frac{\varepsilon}{2}+\delta\right)\left(1+\frac{\varepsilon}{2}\right)+\varepsilon B_{210}\right)+\frac{\left(\frac{\varepsilon}{2}+\delta\right)}{1-\left(\frac{\varepsilon}{2}+\delta\right)}\left(B_{120}+\frac{36}{5 \pi^{2}}\right)
$$

B.3. List of constants and conditions on $\varepsilon$ and $\delta=\delta_{2}$ for Section 5 .

(59)

$$
\begin{aligned}
& \left(1+\frac{\varepsilon}{2}\right) \delta \leq \frac{\pi}{2} \\
& c_{11}=\sup _{\tau \in\left[0, \frac{\pi}{2}\right]}\left|\frac{\tau^{2}+\tau \cos \tau \sin \tau-2 \sin ^{2} \tau}{\tau^{5} \sin ^{2} \tau}-\frac{2}{45 \tau}\right| \\
& c_{12}=\sup _{\tau \in\left[0, \frac{\pi}{2}\right]}\left|\frac{2(\sin \tau-\tau \cos \tau)}{\tau^{3} \sin ^{3} \tau}-\frac{2}{3 \tau^{3}}\left(1+\frac{2 \tau^{2}}{5}\right)\right| \\
& c_{13}=\sup _{\tau \in\left[0, \frac{\pi}{2}\right]}\left|\frac{2(\sin \tau-\tau \cos \tau)}{\tau^{5} \sin \tau}-\frac{2}{3 \tau^{3}}\left(1+\frac{\tau^{2}}{15}\right)\right| \\
& c_{14}=\sup _{\tau \in\left[0, \frac{\pi}{2}\right]}\left|\frac{4\left(\sin ^{2} \tau-\tau^{2}\right)}{\tau^{5} \sin ^{2} \tau}+\frac{4}{3 \tau^{3}}\left(1+\frac{\tau^{2}}{5}\right)\right| \\
& c_{15}=\sup _{\tau \in\left[0, \frac{\pi}{2}\right]}\left|\frac{\tau-\sin \tau}{\tau \sin \tau}\right|, c_{16}=\sup _{\tau \in\left[0, \frac{\pi}{2}\right]}\left|\frac{\tau^{2} \cos \tau-\sin ^{2} \tau}{\tau \sin ^{2} \tau}\right|, \\
& c_{17}=\sup _{\tau \in\left[0, \frac{\pi}{2}\right]}\left|\frac{2 \cos \tau-2+\tau \sin \tau}{\tau^{6}}+\frac{1}{12 \tau^{2}}\right| \\
& \left(1+\frac{\varepsilon}{2}\right) \delta \leq \frac{2}{\sqrt{5}} \\
& C_{2}=\frac{338 C_{1}^{3} \pi^{11}}{8}+\frac{206}{100}\left[8\left(c_{15} c_{6}+c_{16} c_{7}\right)+\left(c_{6}+c_{7}\right) c_{15}\right] \\
& C_{3}=\frac{268 C_{1}^{3} \pi^{11}}{8}+\frac{411}{100}\left[2\left(c_{15} c_{6}+c_{16} c_{7}\right)+\left(c_{6}+c_{7}\right) c_{15}\right] \\
& \text { (61) } \quad \frac{1}{180} \geq \varepsilon\left(C_{2}+19 c_{17} \delta\right)+\frac{115}{100} \delta\left(c_{11}+c_{12}+\frac{1}{2} c_{14}\right) \\
& \frac{1}{180} \geq \varepsilon\left(C_{3}+13 c_{17} \delta\right)+\frac{115}{100} \delta\left(c_{13}+\frac{1}{2} c_{14}\right)
\end{aligned}
$$




\section{B.4. A constant and conditions on $\varepsilon$ for Section 6 .}

$$
\begin{gathered}
C_{4}=338 \pi^{10} C_{1}^{3}+20 \pi\left(c_{6}+c_{7}\right) \\
\varepsilon \leq 2.19 \times 10^{-3} \frac{\sin ^{3} 1}{C_{4}} \delta_{2}^{2} \\
\left(1+\frac{\varepsilon}{2}\right)\left(1+\frac{2 \varepsilon}{\delta_{1}\left(1-\frac{\delta_{1}^{2} \pi^{2}}{96}\right)}\right) \leq \frac{6}{5} \\
\varepsilon \leq \frac{1.3 \times 10^{-4}}{\pi^{3} C_{4}} \sin ^{3}\left(\frac{\pi}{4} \delta_{1}\right)
\end{gathered}
$$

Acknowledgment. We thank Alessio Figalli and Ludovic Rifford for their keen interest in a preliminary draft of this paper (44 pages, communicated to Figalli by Ge on January 14th, 2009). The second author wishes to thank Young-Heon Kim and Robert McCann for stimulating conversations while visiting IPAM at UCLA for a spring workshop on optimal transport (April 2008). Finally, we would like to thank the Referee for a valuable comment.

\section{REFERENCES}

[1] O. Bonnet, Sur quelques propriétés des lignes géodésiques, C. R. Acad. Sci. Paris, 40 (1855), pp. 1311-1313.

[2] Y. BRenier, Polar factorization and monotone rearrangement of vector-valued functions, Comm. Pure Appl. Math., 44 (1991), pp. 375-417.

[3] M. Do CARMo, Differentiable Curves and Surfaces, Prentice-Hall (1976).

[4] M. Do CARmo, Riemannian Geometry, Birkhaüser, Boston (1992).

[5] M. Castelpietra and L. Rifford, Regularity properties of the distance functions to conjugate and cut loci for viscosity solutions of Hamilton-Jacobi equations and applications in Riemannian geometry, ESAIM Control Optim. Calc. Var., 16:3 (2010), pp. 695-718.

[6] J. Cheeger And D. G. Ebin, Comparison Theorems in Riemannian Geometry, North-Holland Math. Library, 9 (1975).

[7] Ph. Delanö̈ and Y. Ge, Regularity of optimal transport on compact, locally nearly spherical, manifolds, J. reine angew. Math., 646 (2010), pp. 65-115.

[8] A. Figalli AND L. Rifford, Continuity of optimal transport maps and convexity of injectivity domains on small deformations of $\mathbb{S}^{2}$, Comm. Pure Appl. Math., LXII:12 (2009), pp. 16701706.

[9] A. Figalli, L. Rifford and C. Villani, On the Ma-Trudinger-Wang curvature on surfaces, Calc. Var., 39:3-4 (2010), pp. 307-332.

[10] A. Figalli, L. Rifford and C. Villani, Nearly round spheres look convex, Amer. J. Math., (to appear).

[11] Y.-H. Kim AND R. J. MCCANn, Continuity, curvature, and the general covariance of optimal transportation, J. European Math. Soc., 12:4 (2010), pp. 1009-1040.

[12] Y.-H. KIM AND R. J. MCCANN, Towards the smoothness of optimal maps on Riemannian submersions and Riemannian products (of round spheres in particular), J. reine angew. Math., (to appear).

[13] P. W. Lee and R. J. MCCann, The Ma-Trudinger-Wang curvature for natural mechanical actions, Calc. Var., 41:1-2 (2011), pp. 285-299.

[14] G. LOEPER, On the regularity of maps solutions of optimal transportation problems, (preprint 08/2006). 
[15] G. Loeper And C. Villani, Regularity of optimal transport in curved geometry: the nonfocal case, Duke Math. J., 151:3 (2010), pp. 431-485.

[16] X.-N. Ma, N. S. Trudinger And X.-J. Wang, Regularity of potential functions of the optimal transportation problem, Arch. Rat. Mech. Anal., 177 (2005), pp. 151-183.

[17] R. J. McCann, Polar factorization of maps on Riemannian manifolds, Geom. Funct. Anal., 11 (2001), pp. 589-608.

[18] J. Milnor, Morse Theory, Princeton Univ. Press, Annals of Math. Stud., 51 (1963).

[19] G. Monge, Mémoire sur la théorie des déblais et remblais, Mémoires de l'Académie Royale des Sciences de Paris (1781).

[20] M. H. Protter And H. F. Weinberger, Maximum Principles in Differential Equations, Prentice-Hall (1967).

[21] C. Villani, Optimal Transport, Old and New, Grundlehren der mathematischen Wissenschaften, 338, Springer-Verlag (2009). 